\title{
A Survey of Interdependent Information Security Games
}

\author{
ARON LASZKA ${ }^{1}$, MARK FELEGYHAZI ${ }^{1}$, LEVENTE BUTTYAN ${ }^{1,2}$ \\ ${ }^{1}$ Laboratory of Cryptography and System Security (CrySyS Lab), Department of Networked \\ Systems and Services (HIT), Budapest University of Technology and Economics (BME) \\ ${ }_{2}^{2}$ MTA-BME Information Systems Research Group, Budapest University of Technology and \\ Economics
}

\begin{abstract}
Risks faced by information system operators and users are not only determined by their own security posture, but are also heavily affected by the security-related decisions of others. This interdependence between information system operators and users is a fundamental property that shapes the efficiency of security defense solutions. Game theory is the most appropriate method to model the strategic interactions between these participants. In this survey, we summarize game-theoretic interdependence models, characterize the emerging security inefficiencies, and present mechanisms to improve the security decisions of the participants. We focus our attention on games with interdependent defenders and do not discuss two-player attackerdefender games. Our goal is to distill the main insights from the state-of-the-art and to identify the areas that need more attention from the research community.
\end{abstract}

Categories and Subject Descriptors: K.6.5 [Management of Computing and Information Systems]: Security and Protection (D.4.6, K.4.2)

General Terms: Security, Economics, Management

Additional Key Words and Phrases: Interdependent security, security economics, security games, externality

\section{INTRODUCTION}

Information security has traditionally been considered a strategic cat-and-mouse game between the defending party and "the attacker". The goal of the attacker has been to compromise the defender's systems and to profit from this unauthorized access, while the goal of the defender has been to prevent unauthorized access to and usage of resources. In this game, both the attacker and the defender have traditionally been focusing on developing new technology to achieve their goals. Especially on the defense side, a traditional approach in information security is to enhance security technologies to reduce the number of vulnerabilities, hence attacks, and their impact on business operation.

Even though the defenses are getting more efficient and protecting more users [Microsoft 2011], the total number of attacks is increasing globally. This trend can mostly be accounted to the increasing number of devices connected to the Internet, and consequently to the increasing interdependence of information systems. Attackers exploit this strong interdependence by launching and operating their attacks on a large-scale from countries where operating costs are reduced and law enforcement is weak. Although the proportion of protected users [Microsoft 2011] is increasing, the equally increasing number of unprotected computer systems leaves ample space to the attackers for exploitation. In addition to interdependence, available security information is highly asymmetric and strongly favors the attackers. A fundamental bias is that attackers only need to exploit one vulnerability of the targeted system, while the defender has to protect as many threat vectors as possible. Attackers can - and often do - proactively test their attack methods offline, but due to the number of attack possibilities, the defenders have a difficult time to patch systems proactively [Anderson 2001]. Moreover, the possibility of using illegal methods gives attackers a broader range of options than defenders. Finally, the "physics" of security changes over time - new classes of attacks are being discovered and this dynamics keeps security researchers and practitioners alert. 
The increasing number of attacks suggests that solely improving information security technologies does not provide adequate protection against the persistent efforts of attackers. The adoption of security defense solutions is rather slow [Ozment and Schechter 2006] and their maintenance overhead often makes them lag behind actual attack trends. There is a growing number of researchers and industry practitioners, who advocate that providing adequate information security requires an economics approach beyond the traditional technology solutions approach [Anderson 2001; Anderson and Moore 2006]. They argue that the main obstacle of adopting information security solutions is the lack of proper incentives for participants to introduce existing solutions, monitor their systems and share relevant information. For another report on the efficiency of existing security solutions, we refer the reader to [Defence Signals Directorate 2012]. The report lists the top 35 mitigation strategies and shows that the top 4 defenses stop more than $85 \%$ of the attacks.

The interaction of a strategic attacker and a defender can be modeled as a game using the mathematical methods of game theory [Gans et al. 2011; Krugman et al. 2008; Mas-Colell et al. 1995]. For example, the interaction between one attacker and one defender can be modeled as a classic two-player game. ${ }^{1}$ Yet, simple two-player games neglect an important aspect of strategic interaction: there are typically several interdependent players on the defender side (and often on the attacker side as well). Interdependent security ${ }^{2}$ games are a natural extension to simple two-player information security games for cases where the defense relies on the efforts of multiple parties. Most of the real-life information security problems correspond to the interdependent security model, and hence the model is a powerful tool to reveal inefficiencies of information security investments. Interdependence is a core property of networked information systems; therefore, it must be considered at the design of information security defense strategies.

In this paper, we survey interdependent security games.

Definition 1.1 (Interdependent Security Game). We define a security game model to belong to the family of interdependent security games if there are multiple selfish but non-malicious players, who can choose whether to invest into security or remain unprotected. Each player's goal is to minimize her own risk, which depends on the investments of some or every other player, and to minimize her security investment costs.

In other words, we do not survey games in which there is only one "defender" (attackerdefender games) or in which the players' risks are independent of the other players' security investments. Note that we survey only those games where the defender's strategic decisions are related to security investments. The effects of malicious behavior on multiple interdependent players have been studied with other strategy spaces as well, for example, in congestion games [Babaioff et al. 2007]. However, due to space limitations, we will not discuss games that do not satisfy Definition 1.1 any further.

In most of the models, the attackers are represented as an exogenous, persistent threat and not as players of the game. Yet, there is evidence that the attacks are the result of the cooperation of various participants from the underground economy [Levchenko et al. 2011]. Clearly, the attackers also play an interdependent attacker game among themselves [Herley and Florêncio 2009; 2010]. This area of se-

\footnotetext{
${ }^{1}$ Several authors consider two-player security games. For a comprehensive survey of two-player security games, we refer the reader to [Manshaei et al. 2013].

${ }^{2}$ In this paper, we will refer to interdependent information security simply as interdependent security, as this naming is widely accepted in the literature and allows for models in a broader context (e.g., physical security on airlines in the seminal paper [Kunreuther and Heal 2003]).
} 
curity modeling is less explored due to the lack of reliable data about the attackers' interactions and credible assumptions about their profit models. Recent large-scale data collection efforts aiming at the understanding of the underground economy point towards this goal [Levchenko et al. 2011]. Our survey focuses on the interdependence of strategic defenders, but we also mention the strategic behavior of malicious attackers when appropriate. Nonetheless, we do not consider games with interdependent attackers. It is worth mentioning that there is surprisingly little work on this topic .

Researchers who surveyed game-theoretic models applied to security problems [Manshaei et al. 2013] typically paid a very limited attention to the problem of interdependence. Interdependence lays at the core of information security problems. The actions of the participants in information systems bear positive and negative effects on others. Understanding these effects and leveraging the acquired expertise could lead to improved security defense solutions. There is a significant body of work on interdependent security games differing in modeling assumptions, solutions approach and arriving at various conclusions. To the best of our knowledge, our survey is the first attempt to summarize the key points of related work. In this survey, we make the following contributions:

- We systematically survey interdependent security game papers to summarize the modeling assumptions and synthesize a common core model with modeling extensions.

- We categorize the equilibrium solutions in interdependent security games, discuss efficiency results, and present how these results change varying key modeling assumptions.

- We summarize solution techniques from related work that aim to improve the security of information systems.

- We present a discussion on research areas that are not well understood and need more attention of the research community.

The paper is organized as follows. First, we introduce basic concepts of market economics in Section 2. We synthesize a core model of interdependent security games in Section 3. In Section 4, we systematize interdependence models in the research literature and connect them to the core model. Section 5 presents extended models, which relax some assumptions of the core model. We discuss classic equilibrium solutions, their efficiency, and related results in Section 6. Section 7 gives an overview of attempts to improve upon the often inefficient equilibria in interdependent security games. Finally, we summarize the work in Section 8 and provide directions for future research.

\section{MARKET ECONOMICS BACKGROUND}

In this section, we give a brief overview of the relevant concepts in economics we use in the paper. The main artifact affecting information security is the existence of externalities as presented in Section 2.1, then we also discuss the role of asymmetric information in Section 2.2 and the effect of monopolies on information security decisions in Section 2.3.

Information security is a public good [Varian 2004; Grossklags et al. 2008] and security defense is organized via market mechanisms and regulation. Since market mechanisms are in place, information security exhibits all the inefficiencies of a free market, but these inefficiencies are magnified by the sensitivity of security information. In particular, information security markets are threatened by causes of classic market failures in economics: externalities of security investment decisions, information asymmetries, and monopoly providers. 
In the following, we briefly summarize these classic economics concepts to allow a reader with a computer engineering background to get familiar with the notions of this paper. For a thorough explanation of these concepts, the reader is referred to a basic textbook on microeconomics such as [Gans et al. 2011; Krugman et al. 2008; Mas-Colell et al. 1995].

\subsection{Externalities of Security Investment Decisions}

In an interdependent market, the actions of the players affect other players. Usually, these actions are captured in the transaction costs of the players, but often the transaction costs do not fully account for the effect of one player's action on others. This "spillover" effect of a player's actions on other players is called an externality. Depending on the nature of the spillover, we can refer to a positive or a negative externality.

In a positive externality, the action of the player has a beneficial effect on herself, but other players also benefit from her investment. Information security defense typically exhibits this type of externality. Information systems rely extensively on networking effects, for example the value of a social network is defined by the number of participants connecting to it. This strong interdependence is often exploited by miscreants to speed up the spread of malware programs and infect a large number of computers [Böhme and Kataria 2006; Anderson and Moore 2006]. In fact, computer crime became really troublesome by the fact that simple attacks can be amplified to a world-wide scale with limited resources. Inherently, security investment of the users or companies prevent the spread of malware infections creating a positive externality for others. Yet, positive externalities have adverse effects. A typical problem is freeriding, when players avoid investing in security expecting other players to protect them. Free-riding significantly contributes to the general under-investment in security as it is observed in real-life.

Conversely, the lack of security investment as an action can be regarded as one having negative externalities. Due to the strong interdependence of information systems, Internet security can be considered as a public good [Varian 2004]. Those who do not care about security are adversely affecting the security of others. Negative externalities are also present when the player protects herself investing in more security defense. A typical example for such an effect is the weakest target game discussed in Section 4.4, where security investment of a player makes her information system more resistant to attacks and this subsequently motivates attackers to choose other targets instead. We note that this substitution effect is difficult to observe as we typically do not possess an in-depth knowledge of the strategic incentives of attackers.

We note that most interdependent security games in related work focus on the case of positive externalities, that is, on the positive effect of security investment decisions as the most important factor influencing security decisions. Negative externalities contribute much less to the security investment decisions of both attackers and defenders, and they are difficult to characterize [Herley and Florêncio 2009; Herley 2010]. We will detail these models in Sections $4.1-4.3$.

\subsection{Asymmetric Information}

The nature of the interaction defines the efficiency of a specific market. The available information on the market participants and the quality of the products and services they offer are key aspects defining market efficiency. It is a well-known result in economics that asymmetric information can cause serious market inefficiencies [Akerlof 1970]. In [Akerlof 1970], Akerlof sketches the classic example of lemon markets in car sales in where low-quality cars (lemons) will drive good-quality cars out of the market if buyers cannot distinguish between the two types. Obviously, sellers have a precise information of the car type, hence the information asymmetry. 
In the security ecosystem, economics and privacy reasons lead to under-reporting of security incidents. This in turn results in a non-transparent market where the efforts of the participants cannot be fairly judged. As a matter of fact, transparency is discouraged because there are other consequences to reporting a security incident. Asymmetric information problems arise in various examples in the information security ecosystem. For example, security products constitute a lemon market themselves because independent evaluation on their provided security is sparse. The certification procedure of security products has inherent weaknesses [Anderson 2001]. As currently the certifiers are contracted by the product developers, adverse incentive effect takes place and as a result products of questionable quality get certified.

Asymmetric information also diminishes the benefits of risk management solutions such as insurance. Cyber-insurance, as it is called for information systems, suffers from the classic insurance artifacts that reduce insurance's efficiency. First, adverse selection exists, because insurance is more beneficial for users with high risk and hence they are more likely to take it. This biased selection of users together with the limited ability of insurance companies to identify the real risk profile of users causes an inefficient allocation of the insurers' resources. Another issue is moral hazard, when the risk perception of users changes when taking insurance. Since the insurance contract shields users from catastrophic events, they are more likely to take higher risks. In information systems, users with anti-virus products are more likely to click on suspicious links expecting the AV product to protect them.

\subsection{Monopoly}

It is well known that monopoly providers can cause inefficiencies in the market as well. The adverse effect of the misaligned incentives in case of a monopoly provider is especially apparent in the security context. A monopoly provider has strong incentives to provide a less than optimal security solution as we discuss in Section 6.6.

Yet, there is an even more serious effect caused by a monopoly provider, that is, the dramatic increase in the correlation of security incidents because a single flaw in the product of a monopoly provider can be exploited at a large number of users [Böhme and Kataria 2006; Anderson and Moore 2006]. In the realm of information security, miscreants are strategic decision-makers themselves and optimize their investments when attacking. To have the most benefit for a unit cost, they tailor their attacks to the software solutions of major providers. A typical example are the attacks against Microsoft products on personal computers due to their dominating presence as an operating system or more recently the rise of Android malware on mobile platforms. We can say that using software from monopoly providers magnifies the exposure of computer systems to attacks and enables large-scale, correlated incidents.

\section{CORE MODEL OF INTERDEPENDENT SECURITY GAMES}

The literature on interdependent security games is very diverse in terms of modeling approaches, assumptions, notations, and solution concepts. In order to be able to discuss the various models in a unified manner, in this section, we synthesize a core model of interdependent security games and introduce a common notation. A very important element of this core model, the model of the interdependence between players, will be discussed separately in Section 4 due to its complexity. Then, in Section 5, we present various extensions to the core model, which relax certain assumptions.

\subsection{Notations and Notational Conventions}

We summarize the notations used in this paper in Table I. Vectors are assumed to be column vectors and denoted by bold symbols (e.g., $\boldsymbol{x}=\left[x_{1}, \ldots, x_{N}\right]^{T}$ is the vector of 
Table I. Notations Used in the Paper

\begin{tabular}{|c|c|c|}
\hline Symbol & Description & \\
\hline \multicolumn{3}{|c|}{ Core Model } \\
\hline$N$ & number of players & \multirow{5}{*}{ of / for player $i$} \\
\hline$x_{i}$ & security investment & \\
\hline$f_{i}$ & risk function & \\
\hline$C_{i}$ & (unit) cost of security investment & \\
\hline$L_{i}$ & loss when compromised & \\
\hline \multicolumn{3}{|c|}{ Interdependence Models \& Extensions } \\
\hline$B$ & number of byzantine players & \multirow{7}{*}{ of / for player $i$} \\
\hline$\alpha$ & risk non-additivity & \\
\hline$F$ & friendship factor & \\
\hline$W_{i}$ & initial wealth (or endowment) & \\
\hline$d_{i}$ & number of neighbors & \\
\hline$\omega_{i j}$ & influence of player $j$ on player $i$ & \\
\hline$\tau_{i j}$ & rate of traffic from player $i$ to player $j$ & \\
\hline
\end{tabular}

Note: When a value is uniform over the set of players, we omit the subscript (e.g., if the unit cost of investment is the same for all players, we let $C$ denote it).

security investments). When a value is uniform over the set of players, we omit the subscript $i .^{3}$

\subsection{Core Model}

There are $N$ interconnected players, who are assumed to be selfish but non-malicious, while attackers are modeled as exogenous threats. The players are also generally assumed to possess complete information and to be rational and risk-neutral ${ }^{4}$.

The security investment of player $i$ is denoted by $x_{i}$, and it can be modeled both as discrete (e.g., $x_{i}=0$ if player $i$ does not invest and $x_{i}=1$ if player $i$ invests ${ }^{5}$ ) and continuous (i.e., $x_{i} \in \mathbb{R}_{\geq 0}$ ). Discrete investments can model, for example, the purchase of a security product, such as an antivirus software. An example for continuous investment decisions is setting the sensitivity of security monitoring systems (IDS). In this latter case, higher sensitivity of the security monitoring system generates more alarms and warnings, which need to be processed by security experts incurring significant costs. Discrete investments are assumed, for example, in [Kunreuther and Heal 2003; Lelarge and Bolot 2008; Grossklags et al. 2008] and in all of the games that are based on the inoculation interdependence model ${ }^{6}$. Continuous investments are assumed, for example, in [Varian 2004; Jiang et al. 2011; Böhme 2012]. The discrete investment assumption does not necessarily have to be a restriction. For example, in [Grossklags et al. 2010a], discrete investments are assumed, but sensitivity analysis with respect to the discrete choice assumption shows that differences between the discrete and continuous cases arises only in some boundary cases of limited practical relevance.

The risk of an incident, such as a security breach, for player $i$ depends on the investment of player $i$ as well as the investments of the other players. The value of player $i$ 's

\footnotetext{
${ }^{3}$ Please note the difference in notation between vectors and uniform constants. For example, $C$ denotes the unit cost of investment for every player, while $\boldsymbol{C}$ denotes the vector consisting of each player's unit cost of investment.

${ }^{4}$ Much of the economic conflict literature related to production, appropriation, defense, and rent seeking also assumes risk neutrality [Hausken 2006].

${ }^{5}$ We note here that in the vast majority of research papers, discrete investment modeling means a binary decision.

${ }^{6}$ The inoculation model is introduced in Section 4, along with the other interdependence models.
} 
risk is computed using a risk function $f_{i}$ as

$$
f_{i}(\boldsymbol{x})=f_{i}\left(x_{i}, \boldsymbol{x}_{-i}\right),
$$

where $\boldsymbol{x}_{-i}$ is the investment vector of all players but player $i$. The risk function $f_{i}$ is often assumed to be the probability of a security incident, in which case $f_{i} \in[0,1]$. The exact form of the risk function is determined by the model of interdependence between the players. In the literature, various models of interdependence have been proposed, which we will discuss in Section 4 . For now, we only assume that $f_{i}$ is non-decreasing in $x_{i}$ for every player $i$.

The goal of player $i$ is to maximize her expected payoff, which is defined as

$$
-L_{i} f_{i}(\boldsymbol{x})-C_{i} x_{i}
$$

where $L_{i}$ is the potential loss if an incident indeed occurs and $C_{i}$ is the (unit) cost of investment for player $i .^{7}$ Equivalently, each player $i$ can minimize her expected cost, which is

$$
L_{i} f_{i}(\boldsymbol{x})+C_{i} x_{i} .
$$

The risk of a player is often decomposed into two parts: direct risk and indirect risk (e.g., [Kunreuther and Heal 2003; Kearns and Ortiz 2004; Lelarge and Bolot 2008]). Almost without exception in the literature, risks are assumed to be non-additive, that is, a player can sustain either direct or indirect loss, but not both. In [Heal and Kunreuther 2004], a risk non-additivity parameter $\alpha$ is introduced, which measures the extent to which losses are non-additive. If $\alpha=0$, then the total risk of a player is the sum of her direct and indirect risks; if $\alpha=1$, then indirect losses are conditioned on the direct losses not occurring.

In discrete security investment models, perfect protection (also called complete or strong protection) is frequently assumed, which means that a player's overall risk is always zero when she invests in security. ${ }^{8}$ Examples of models assuming perfect protection include the model of [Lelarge and Bolot 2008], the second class of problems in [Heal and Kunreuther 2004], the model of [Theodorakopoulos et al. 2013], and every inoculation game. It is also often assumed that the probability of direct loss is zero when a player invests in security, e.g., in [Kunreuther and Heal 2003; Kearns and Ortiz 2004; Heal and Kunreuther 2004].

In classic epidemic models ${ }^{9}$, it can also be assumed that there is no direct risk at all, only indirect; for example, in [Omic et al. 2009; Theodorakopoulos et al. 2013]. Perfect or strong protection can be assumed in this case as well; for example, in [Theodorakopoulos et al. 2013].

\section{MODELS OF INTERDEPENDENCE}

In this section, we systematize the models proposed in the literature for interdependence between players. Recall that, based on the players' security investments $x$, a model of interdependence determines each player $i$ 's risk, which we represented as a general function $f_{i}(\boldsymbol{x})$ in the previous section. In this section, we provide a classification of interdependence models (see Table II), and describe each one in more detail.

The primary interdependence between players is that security investments create positive externalities (as discussed in Section 2.1). Positive externality means that the investments of other players have a positive effect on the security and, consequently,

\footnotetext{
${ }^{7}$ Many papers assume that the potential loss is 1 for every player or, alternatively, that the (unit) cost is 1 , and incorporate the ratio between loss and cost into $C_{i}, L_{i}$ or $f_{i}$.

${ }^{8}$ Recall that, in discrete security investment models, binary investment is assumed predominantly.

${ }^{9}$ Classic epidemic models are introduced in Section 4, along with the other interdependence models.
} 
Table II. Summary of Models of Interdependence Between Players

\begin{tabular}{|c|c|c|c|}
\hline & Model & Externalities & Related work \\
\hline \multirow{2}{*}{\multicolumn{2}{|c|}{ general }} & positive & $\begin{array}{l}\text { [Gordon et al. 2003] [Ogut et al. 2005] } \\
\text { [Jiang et al. 2011] }\end{array}$ \\
\hline & & both & [Heal and Kunreuther 2004] \\
\hline \multirow[b]{2}{*}{ propagation } & epidemic & \multirow{4}{*}{ positive } & $\begin{array}{l}\text { [Lelarge and Bolot 2008] [Lelarge } \\
\text { 2009] SIS [Omic et al. 2009] SIP } \\
\text { [Theodorakopoulos et al. 2013] }\end{array}$ \\
\hline & inoculation & & $\begin{array}{l}\text { [Aspnes et al. 2004] [Moscibroda et al. } \\
\text { 2006] [Meier et al. 2008] [Díaz et al. } \\
\text { 2009] [Kumar et al. 2010] }\end{array}$ \\
\hline \multirow{8}{*}{ other } & weakest link & & $\begin{array}{l}\text { [Varian 2004] [Grossklags et al. 2008] } \\
\text { [Grossklags et al. 2010a] [Grossklags } \\
\text { et al. 2010b] }\end{array}$ \\
\hline & best shot, total effort & & $\begin{array}{l}\text { [Varian 2004] [Grossklags et al. 2008] } \\
\text { [Grossklags et al. 2010a] [Grossklags } \\
\text { et al. 2010b] [Pal and Hui 2011] }\end{array}$ \\
\hline & linear & both & $\begin{array}{l}\text { linear influence [Miura-Ko et al. } \\
\text { 2008b] [Miura-Ko et al. 2008a] [Saad } \\
\text { et al. 2010] [Radosavac et al. 2008] ef- } \\
\text { fective investment [Jiang et al. 2011] }\end{array}$ \\
\hline & $\begin{array}{l}\text { stochastic one-hop } \\
\text { propagation }\end{array}$ & \multirow{2}{*}{ positive } & $\begin{array}{l}\text { discrete [Kunreuther and Heal 2003] } \\
\text { [Kearns and Ortiz 2004] [Heal and } \\
\text { Kunreuther 2005] continuous [Böhme } \\
\text { 2012] }\end{array}$ \\
\hline & other & & $\begin{array}{l}\text { bad traffic [Jiang et al. 2011] net- } \\
\text { worked control systems [Amin et al. } \\
\text { 2012] [Amin et al. 2011] }\end{array}$ \\
\hline & strategic adversary & \multirow[b]{2}{*}{ both } & [Hausken 2006] \\
\hline & $\begin{array}{l}\text { stochastic one-hop } \\
\text { with adversary }\end{array}$ & & [Ceyko et al. 2011; Chan et al. 2012] \\
\hline & weakest target & negative & [Grossklags et al. 2008] \\
\hline
\end{tabular}

the payoff of a player, while negative externality means the contrary. This positive externality can be explained in many ways: a successfully compromised player can be used to mount attacks against players that depend on it, investments of a single players can result in security patches that can be used by every other player, etc. However, a player's investment can also have a negative externality on other players. Security investment of a user causes her to become a less attractive target for the adversaries and, consequently, the adversaries spend more of their resources on attacking other players [Hausken 2006].

The organization of this section is the following. First, in Section 4.1, we discuss general models, which do not assume some specific attack mechanism to explain the interdependence between the players and, hence, have only some mild constraints on $f_{i}(\boldsymbol{x})$. Then, in Section 4.2, we discuss models which assume that interdependence is caused by the propagation of security incidents and compromises. ${ }^{10}$ Next, in Section 4.3 , we discuss models which focus on positive externalities and assume some specific (but not propagation-based) mechanism to explain interdependence. Finally, in Section 4.4, we discuss models which focus on negative externalities.

\footnotetext{
${ }^{10}$ In other words, these propagation-based models assume that once an attacker or malware compromises a player, it will be able to compromise the neighbors more easily.
} 


\subsection{General Models}

It is possible to derive results from general models of interdependence, in which the risk function $f$ can be an arbitrary function that satisfies a set of assumptions.

The most common assumption is that security investments exhibit positive but declining returns for every player [Gordon et al. 2003; Ogut et al. 2005; Jiang et al. 2011]. The positive returns (i.e., strictly decreasing risks) model the positive externalities between the players: if one player increases her investment in security, every player benefits. Formally, $\frac{\partial f_{i}(\boldsymbol{x})}{\partial x_{j}}<0, \forall i, j$. The declining returns (i.e., convexity of the risk function) model the diminishing marginal utility of security investments, a generally accepted assumption. Formally, $\frac{\partial^{2} f_{i}(\boldsymbol{x})}{\partial x_{j}^{2}}>0, \forall i, j$.

The target set of the risk function is also often restricted. In [Ogut et al. 2005], the risk function $f_{i}$ is assumed to measure the probability of an incident at player $i$; consequently, it has to satisfy $f_{i}(\boldsymbol{x}) \in[0,1], \forall i$. In the general model of [Jiang et al. 2011], the risk function has no such meaning and it is only required to be finite and to satisfy $f_{i}(\mathbf{0})>0, \forall i$.

In [Gordon et al. 2003], such a general two-player model is introduced to study security-based information sharing organizations (SB/ISOs), but which can also be used to model positive externalities arising from a wide-range of other types of interactions between the players. ${ }^{11}$

In [Heal and Kunreuther 2004], a slightly less general model is presented, which decomposes risk into direct and indirect parts. The expected indirect loss of player $i$, when she follows strategy $x_{i} \in\{S, N\}$ and the players in the set $K$ invest in security, is denoted by $q_{i}\left(K, x_{i}\right)$. Then, the expected cost of investing is $c_{i}+q_{i}(K, S)$, where $c_{i}$ is the cost of the security investment, while the expected cost of not investing is $p_{i} L_{i}+\left(1-\alpha p_{i}\right) q_{i}(K, N)$, where $\alpha \in[0,1]$ measures the extent to which damages are non-additive and $p_{i}$ is the probability of a direct loss for player $i$. The model is used to study three classes of problems:

- Partial protection: $q_{i}(K, N)=q_{i}(K, S)$ and $\alpha=1$, so that $c_{i}(K)=p_{i}\left(L_{i}-q_{i}(K, N)\right)$, where $c_{i}(K)$ is the cost of investment at which player $i$ is indifferent between investing and not investing. Observe that $c_{i}(K)$ is increasing in $K$, that is, the more players invest in security, the more likely it is that others will follow. In this class, a player's investment reduces both her own risk and the risk experienced by other players. This class can be used to model, for example, airline baggage security.

-Complete protection: $q_{i}(K, S)=0$ and $\alpha=1$, so that $c_{i}(K)=q_{i}(K, N)\left(1-p_{i}\right)+$ $p_{i} L_{i}$. Contrary to the previous class, the threshold cost $c_{i}(K)$ is now decreasing in $K$, which means that the more player invest in security, the less likely it is that others will invest as well. In this class, if a player invests in security, then she cannot be harmed at all by the actions or inactions of others. This class can be used to model, for example, a completely effective vaccine against a contagious disease.

- Positive externalities: $q_{i}(K, N)=q_{i}(K, S)$, so that $c_{i}(K)=p_{i}\left(q_{i}(K, S)-L_{i}\right)$. Note that, similarly to the previous class, the threshold cost $c_{i}(K)$ is decreasing in $K$. In this class, an investment by one player creates positive externalities, making it less attractive for others to follow. This class can be used to model, for example, firms' decisions on research and development (R\&D) expenditures.

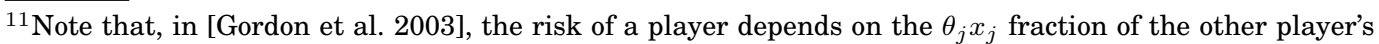
investment $x_{j}$. The sharing portion $\theta_{j}$ is discussed in detail in Section 7.2.8, until then we can assume that it is incorporated into the general function $f$.
} 


\subsection{Attack Propagation Models}

Propagation-based models are motivated by the idea that a player's risk usually does not depend directly on her peers' investment levels. For example, the direct cause of receiving a computer worm via e-mail is the sender's computer being infected with the worm; hence, a player's risk of receiving a worm depends directly on her peers' risks, not their investment levels. The peers' investment levels affect a player's risk indirectly, since they affect the peers' risks directly (e.g., they affect the peers' risk of being infected with the worm). Note that this idea fits in the core model perfectly, as player $i$ 's risk $f_{i}(\boldsymbol{x})$ depends on the investment levels $\boldsymbol{x}$ ultimately.

As a motivating example, consider popular software products that are run at a significant number of devices. Due to market dynamics, these products create a quasimonopolistic situation (introduced in Section 2.3), which is the case with Microsoft Windows in the realm of desktop operating systems, and which is becoming the case with Android for mobile devices. This monopolistic market situation allows malicious software to spread to a large number of devices, which is often modeled using propagation-based attack models.

4.2.1. Epidemic Models. Epidemic models describe how a transmittable disease spreads or extinguishes in a network of individuals. These models can readily be applied in the study of information security, for example, to model viruses spreading in computer networks. If the virus protection (or recovery) decisions of the individuals are modeled using game theory, the resulting model is a propagation-based interdependent security game. In this case, the security investment decisions correspond to the virus protection decisions, and the risk of a player is the risk of being infected.

In an epidemic model, each player at any given moment in time can be in one of the states that represent different stages of the epidemic. The most commonly used states are susceptible, which denotes players who are not infected, but are susceptible to the virus, and infected, which denotes players who are infected and capable of spreading the virus to susceptible players. The transitions between these states are usually modeled as stochastic processes, which are controlled by the investment decisions of the players. For example, the probability of a susceptible player becoming infected within a certain time period can depend on her security investment and the number of infected players that she is connected to.

In the SIS (Susceptible Infected Susceptible) model, there are only two states, susceptible and infected. In this model, infected players are eventually cured of the disease, and then become susceptible immediately. In [Omic et al. 2009], an $N$ interwined SIS model based game is proposed. The $N$-interwined model is an analytically tractable SIS model, which makes only one approximation of a mean-field kind, and whose accuracy improves as the size of the network increases [Mieghem et al. 2009]. In the proposed game-theoretic model, each player's security investment decision determines her curing rate. More specifically, the transition of a player from the infected state to the susceptible state is determined by a Poisson process whose rate is equal to the player's investment.

The SIP (Susceptible Infected Protected) model presented in [Theodorakopoulos et al. 2013] introduces a protected state, which represents players who invest in security and, therefore, are immune to the virus. Players in the susceptible or protected state occasionally learn the state of the network and have an opportunity to revise their current investment decisions, that is, they can choose between being susceptible or being protected. Players in the infected state are eventually disinfected and, then, become protected. All of these opportunities and transitions are modeled as Poisson processes. 
As the number of players increases, propagation based models can become very complex. One way to cope with this complexity is to use mean-field approximation [Theodorakopoulos et al. 2013]. In this case, instead of following each player's state, only the number of players in each state is kept track of, which allows the transition functions to be expressed as deterministic functions of the system state.

In [Lelarge and Bolot 2008; Lelarge 2009], local mean-field (LMF) analysis is proposed, which extends mean-field approximation by allowing to model the correlation structure on local neighborhoods in the network. It is shown that LMF gives exact asymptotic results as the number of players tends to infinity for sparse random network graphs with a given degree distribution. In [Lelarge and Bolot 2008; Lelarge 2009], LMF is used to study a propagation-based model, in which players can be either infected directly (i.e., direct loss) or indirectly through their infected neighbors (i.e., indirect loss). The probabilities of direct loss and contamination from an infected neighbor are determined by the investment decision of the player.

4.2.2. Inoculation Games. One of the most prevalent propagation based model for interdependence is the inoculation game, which was introduced by Aspnes et al. in [Aspnes et al. 2004].

In the basic inoculation game [Aspnes et al. 2006], the players correspond to the nodes of an undirected graph $G=(V, E)$. Investment decisions are discrete: if $x_{i}=0$, player $i$ remains unprotected; if $x_{i}=1$, player $i$ inoculates herself and she is considered secure. After the players made their choices, the adversary picks some node uniformly at random as a starting point for an infection. The infection then propagates through the graph, infecting a node if she is unprotected and any of her neighbors becomes infected. In the basic model, the cost being secure and the cost of being infected are both uniform.

In the model of [Moscibroda et al. 2006], which we will discuss in Section 5.3, the inoculation game is extended by allowing some players to be malicious or byzantine rather than selfish. In [Meier et al. 2008], which we discuss in Section 5.2, the players also take the costs of their neighbors into account by a factor $F$, called the friendship factor. In [Díaz et al. 2009], the basic inoculation game is used to study the question whether a mediator can increase social welfare by implementing a correlated equilibrium, which is discussed in Section 7.1.3. In [Kumar et al. 2010], the inoculation game is generalized by allowing arbitrary security and infection costs, and arbitrary distributions for the starting point of the infection. More significantly, the generalized inoculation game includes a network locality parameter $l$ that represents a hop limit on the spread of the infection.

\subsection{Other Models Focusing on Positive Externalities}

In this subsection, we discuss the remaining models of interdependence with positive externalities, which assume some specific but not propagation-based mechanism between the players. Note that, even though some of them model how an incident propagates from one player to another (e.g., [Kunreuther and Heal 2003]), we do not consider them to be propagation-based, since they do not allow incidents to spread farther than one hop. In other words, if a model assumes propagation, a player's risk is still influenced only by those players to which she is directly connected.

In [Varian 2004], three prototypical interdependence models are introduced: weakest link, best shot, and total effort. These models are based on the idea that security is a public good; hence, each player's risk is determined by the overall level of security. More formally, in all three of these models, the probability of successful operation is $P(H(\boldsymbol{x}))$ for every player, where $P$ is a differentiable, monotonically increasing and concave function, and $H$ depends on which model is used (see below). The function $P$ is 
often assumed to be a linear mapping, which simplifies to the identity function if $x_{i} \in$ $[0,1]$ for every $i$ (e.g., in [Grossklags et al. 2008; Grossklags et al. 2010a; Grossklags et al. 2010b]). The three models are defined as follows.

- In the weakest link (also called perimeter defense) model, the level of security is determined by the smallest security investment. Formally,

$$
H(\boldsymbol{x})=\min _{i} x_{i} .
$$

Weakest link interdependence can be used to model, for example, the perimeter defenses of enterprises, which are vulnerable if an attacker can identify a weakness that leads to their circumvention. This tightly coupled dependency can be modeled by considering the minimum investment [Grossklags et al. 2008; Grossklags et al. 2010a; Grossklags et al. 2010b].

- In the best shot model, the level of security is determined by the largest security investment. Formally,

$$
H(\boldsymbol{x})=\max _{i} x_{i} .
$$

Best shot interdependence can be used to model security scenarios with built-in redundancy, for example, censorship-resistant networks, where a piece of information is available to the players as long as at least one of them is secure [Pal and Hui 2011; Grossklags et al. 2008; Grossklags et al. 2010a].

- In the total effort (also called cumulative defense and sum-of-efforts) model, the level of security is determined by the sum of the security investment of all players. Formally,

$$
H(\boldsymbol{x})=\frac{1}{N} \sum_{i} x_{i}
$$

Total effort interdependence is used to model the security of end users, who are subject to cumulative interdependencies. For example, an under-investing user who causes increased spam activity represent a security risk to every other user [Grossklags et al. 2008; Grossklags et al. 2010a; Grossklags et al. 2010b; Pal and Hui 2011].

The total effort model is appealing as it is relatively simple, yet it can be used to study a wide range of phenomena, such as free-riding. However, it is based on the assumption that each player's risk is influenced uniformly by every other player, which severely limits its application. In many practical security problems, interdependence relations are nonuniform or infrequent: individual users receive e-mails from only a subset of all the users in a system, firms only do business with a set of partners, etc.

The model can generalized by replacing the summation with an arbitrary linear combination. In the linear influence model introduced in [Miura-Ko et al. 2008b], the linear combination is expressed using a weight matrix $\Omega$, where $\omega_{i j}$ is the degree of player $j$ 's influence on player $i$. Then, the risk of player $i$ is

$$
P_{i}\left(\boldsymbol{\omega}_{i}\right),
$$

where the subscript $i$ of $P_{i}$ signifies that $P_{i}$ also depends on the identity of the player. Note that the above formula can also incorporate direct risks if the $\omega_{i i}$ elements of the matrix are filled in accordingly. In [Saad et al. 2010], the linear influence model of security investments is complemented with an additional linear network, which models how much the vulnerabilities of one player influence or threaten the other players. In this model, the payoff of a player is the difference between the positive and negative influences that are caused by the security investments and the vulnerabilities 
of the neighboring players. The linear influence model is also used in [Radosavac et al. 2008] and [Miura-Ko et al. 2008a].

A similar model, called effective investment, is presented in [Jiang et al. 2011]. Let $\omega_{i j}$ measure the "importance" of player $j$ to player $i$. Then, the total risk of player $i$ is

$$
L_{i} P_{i}\left(\sum_{j=1}^{N} \omega_{i j} x_{j}\right) \text {. }
$$

Besides the above classic models of interdependence, several other models have been proposed, which are usually tailored to more specific information security problems:

- In [Kunreuther and Heal 2003] and [Kearns and Ortiz 2004], stochastic one-hop propagation models are introduced, which can be applied to a wide range of security problems, such as airline baggage security, fire safety, or computer viruses. To model indirect risks, let $q_{j i}$ denote the probability that player $i$ is harmed as a result of player $j$ not investing in security. To compute the probability that player $i$ is harmed, assume that risks are non-additive and that security decisions are binary. Then, the total risk of player $i$ is

$$
\left(1-x_{i}\right) p_{i} L_{i}+\left(1-\left(1-x_{i}\right) p_{i}\right)\left[1-\prod_{j \neq i}\left(1-\left(1-x_{j}\right) q_{j i}\right)\right] L_{i},
$$

where $p_{i}$ is the direct risk probability of player $i$ [Kunreuther and Heal 2003; Kearns and Ortiz 2004]. ${ }^{12}$

- In [Jiang et al. 2011], another model of interdependence is introduced besides the general and the effective investment models, which we have discussed previously. The bad traffic model is based on the amount of malicious traffic (e.g., traffic that causes virus infection) from one player to another. Clearly, the security risk posed by a unit of traffic depends on the investments of both players, so the probability that a unit of traffic from player $k$ harms player $i$ can be denoted by $\phi_{k, i}\left(x_{k}, x_{i}\right)$. Then, the rate at which player $i$ is harmed by traffic from player $k$ is $\tau_{k i} \phi_{k, i}\left(x_{k}, x_{i}\right)$, where $\tau_{k i}$ is the rate of traffic from $k$ to $i$, and the total risk of player $i$ is

$$
L_{i} \sum_{k \neq i} \tau_{k i} \phi_{k, i}\left(x_{k}, x_{i}\right) \text {. }
$$

If the security investment is implemented as a traffic filter (e.g., a firewall) and this filter is symmetric (i.e., treats incoming and outgoing traffic in the same way), then it can be assumed that $\phi_{k, i}\left(x_{k}, x_{i}\right)=\phi_{i, k}\left(x_{i}, x_{k}\right)$.

- In [Amin et al. 2011], a special interdependence model is proposed for networked control systems (NCSs), which generalizes the model of [Amin et al. 2012]. The problem of the security choices of individual NCS is formulated as a two-stage game, in which players make their security and control decisions, respectively. Each player's plant is modeled as a discrete-time stochastic system, which is controlled by the input sequence chosen in the second stage. The model incorporates both reliability and security risk; the latter reflects the interdependence among players due to their systems being networked.

- In [Böhme 2012], a two-player model is introduced to study the effectiveness of audits. The functional relationship between security investment $x_{i}$ and the probability

\footnotetext{
${ }^{12}$ In [Kunreuther and Heal 2003], the model is first introduced for airline baggage security, where an unprotected player can "contaminate" only one other player, and it is later adapted to computer security, which results in the above model.
} 
$p_{i}\left(x_{i}\right)$ of a direct loss occurring is adopted from the Gordon-Loeb model [Gordon and Loeb 2002]. Formally, $p_{i}\left(x_{i}\right)=\beta^{-x_{i}}$, where $\beta$ is the player-specific security productivity. The probability that either direct or indirect loss occurs is computed in the same way as in the stochastic one-hop models of [Kunreuther and Heal 2003] and [Kearns and Ortiz 2004]:

$$
f_{1}\left(x_{1}, x_{2}\right)=1-\left(1-\beta^{-x_{1}}\right)\left(1-\delta \beta^{-x_{2}}\right)
$$

where $\delta$ is the degree of interdependence.

\subsection{Models Focusing on Negative Externalities}

Negative externalities ${ }^{13}$, introduced in Section 2.1, created by the players' investments do not rely on explicit interdependence relationships between the players, thus they are fairly difficult to model. More precisely, it is very hard to characterize the set of affected players and estimate the strategic moves of an attacker after a player hardens her defense. This is probably the main reason why there is a limited literature studying this issue.

Yet, there are a few attempts to incorporate negative externalities into interdependent security models. In [Heal and Kunreuther 2004], negative externalities are modeled by assuming that the probability of direct loss for a non-investing player, which is constant in the basic model, increases as the number of investing players grows.

In [Hausken 2006], negative externalities are modeled by introducing an adversarial player, who considers the players' strategies and substitutes into the most optimal attack allocation. The adversary invests an amount of $X$ with a unit cost of $C$ into attacking the players. The fraction of the attack directed at player $i$ is $X_{i}$, where $\sum_{i=1}^{N} X_{i}=X$. The attack on player $i$ is assumed to take a form that is common in the conflict and rent seeking literature, where player $i$ keeps a fraction $h_{i}$ of her initial wealth $W_{i}$, while the adversary gets the remaining fraction $1-h_{i}$, where $h_{i}$ is the contest success function. In [Hausken 2006], the common ratio formula is used for $h_{i}$ :

$$
h_{i}=\frac{x_{i}}{x_{i}+X_{i}} .
$$

Consequently, the payoff of player $i$ is

$$
\frac{x_{i}}{x_{i}+X_{i}} W_{i}-C_{i} x_{i}
$$

and the payoff of the adversary is

$$
\sum_{i=1}^{N} \frac{X_{i}}{x_{i}+X_{i}} W_{i}-C X .
$$

For analytical tractability, the model is based on a two-stage game. Both orders of decisions making are studied, that is, both when the adversary moves first and the other players move second and vice versa.

In [Grossklags et al. 2008], two models with negative investment externalities are introduced.

- In the weakest target model, the attacker is always able to compromise the player(s) who invests the least, but leaves the other players unharmed. This models an attacker who has infinite strength and is determined to compromise an arbitrary set of players with the lowest possible effort.

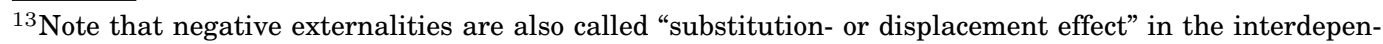
dent security literature. We use the above nomenclature to avoid confusion with the classic notion of the substitution effect in economics.
} 
Table III. Summary of Modeling Assumptions in Related Work

\begin{tabular}{|c|c|}
\hline Assumption & Related work \\
\hline Investment decision discrete & $\begin{array}{l}\text { [Kunreuther and Heal 2003; Kearns and Ortiz 2004; } \\
\text { Heal and Kunreuther 2004; Aspnes et al. 2004; Heal } \\
\text { and Kunreuther 2005; Moscibroda et al. 2006; Meier } \\
\text { et al. 2008; Lelarge and Bolot 2008; Grossklags et al. } \\
\text { 2008; Grossklags et al. 2010a; Lelarge 2009; Díaz et al. } \\
\text { 2009; Kumar et al. 2010; Amin et al. 2012; Theodor- } \\
\text { akopoulos et al. 2013; Amin et al. 2011] }\end{array}$ \\
\hline continuous & $\begin{array}{l}\text { [Varian 2004; Ogut et al. 2005; Hausken 2006; } \\
\text { Grossklags et al. 2008; Miura-Ko et al. 2008b; Miura- } \\
\text { Ko et al. 2008a; Radosavac et al. 2008; Omic et al. } \\
\text { 2009; Jiang et al. 2011; Pal and Hui 2011; Böhme } \\
\text { 2012] }\end{array}$ \\
\hline Incomplete information & $\begin{array}{l}\text { only the distribution of the other players' direct } \\
\text { threats is known [Grossklags et al. 2010a], only the } \\
\text { distribution of the degrees of one's neighbors is known } \\
\text { [Pal and Hui 2011] }\end{array}$ \\
\hline Non-rational \& altruistic players & $\begin{array}{l}\text { non-strictly rational players [Theodorakopoulos et al. } \\
\text { 2013], altruistic players [Meier et al. 2008] }\end{array}$ \\
\hline Malicious players & $\begin{array}{l}\text { strategic adversary [Hausken 2006], byzantine play- } \\
\text { ers [Moscibroda et al. 2006] }\end{array}$ \\
\hline Risk-averse players & $\begin{array}{l}\text { utility function [Ogut et al. 2005; Lelarge and Bolot } \\
\text { 2008] }\end{array}$ \\
\hline
\end{tabular}

- The weakest target with mitigation model is a variation of the weakest target model. The difference is that the probability of a successful attack on the player(s) who invest the least depends on their investment level in this model. This models an attacker who has finite strength.

In [Ceyko et al. 2011] and [Chan et al. 2012], the stochastic one-hop propagation model of [Kunreuther and Heal 2003] is extended to account for strategic attacks, which take the players' security investments into account. In particular, the attacker is modeled as a strategic player, who can choose for each player whether to launch an attack or not. The attacker's goal is to maximize the sum of the players' costs while minimizing the number of attacks she has to launch.

Finally, note that linear interdependence models can also incorporate negative externalities through negative degrees of influence or importance. Examples of such models are the linear influence model in [Miura-Ko et al. 2008b] and the effective investment model in [Jiang et al. 2011], which are discussed in Section 4.3.

\section{EXTENSIONS TO THE CORE MODEL OF INTERDEPENDENT SECURITY GAMES}

In the core model, we assumed all players to possess complete information and to be rational, selfish, non-malicious, and risk-neutral. However, certain papers relax these assumptions to allow for more realistic modeling. In this section, we present these as extensions to the core model, which relax some assumptions to allow for incomplete information (Section 5.1), non-rational players (Sections 5.2), malicious players (Section 5.3), and risk-averse players (Section 5.4). In Table III, we present key papers from related work and the most significant modeling assumptions they make. Results on how these extensions affect the game will be presented in Section 6 after the general results.

\subsection{Incomplete Information}

In practice, individuals rarely possess complete information about the situation they are acting in. This limitation is especially true in the context of security, where the 
adversarial threat is almost always unknown and the effectiveness of security investments, such as firewalls, is very hard to measure.

In [Grossklags et al. 2010a], the maximum discrepancy in the expected payoff of an expert player in a complete information environment versus in an incomplete information environment is studied. The expert player is assumed to possess superior technical and structural understanding of computer security threats and defense mechanisms. Therefore, she correctly understands how her utility is computed, based on the interdependencies that exists in the network. In a complete information environment, the expert player knows the actual direct attack probabilities of all players. In an incomplete information environment, on the other hand, the expert player knows only the probability distribution of the other players' direct attack probabilities and the actual value of her own direct attack probability. In both environments, all the other players are modeled as non-expert players, who underappreciate the interdependence of network security and try to optimize a perceived utility, which actually differs from realized utility.

In [Pal and Hui 2011], the authors study the equilibrium behavior of players who possess only partial information about their underlying neighborhood connectivity structures. Each player $i$ is assumed to know her own degree $d_{i}$ (i.e., the number of other players to whom she is connected somehow), but has information regarding only the probability distributions of her neighbors' degrees $\boldsymbol{d}_{\mathcal{N}_{i}}$, i.e., knows the values of $P\left(\boldsymbol{d}_{\mathcal{N}_{i}} \mid d_{i}\right)$. The players are assumed to begin with ex-ante symmetrical beliefs and common priors regarding the degrees of their neighbors, which are then updated based on their own degrees. Each player is also assumed to be aware of the degree correlation between the neighboring nodes and to account for it when deciding on her strategy. The strategic interactions are modeled as a Bayesian game of incomplete information, whose type space is the player knowledge on the potential degrees of her neighbors.

\subsection{Non-Rational and Altruistic Players}

The assumptions of strict rationality and pure selfishness are very rough simplifications compared to reality. In practice, individuals often make non-rational decision and respect the interests of their peers.

In [Theodorakopoulos et al. 2013], non-strictly-rational players are introduced into a game based on an epidemic interdependence model. The stability and the domains of attraction of the game's equilibria are studied in three scenarios: homogeneous strictly rational players, homogeneous non-strictly rational players, and strictly rational players who are divided into two response classes (i.e., players are grouped together based on their behavior). In the first scenario, players always make investment decisions that minimize their expected costs. In the second, non-strictly rational scenario, the players' investment decisions are suboptimal, but as the level of threat increases, the probability that a player invests in security increases monotonically. In the third scenario, the players are strictly rational, but inhomogeneous: they are divided into two classes, which correspond to different loss values and costs of investment.

In [Meier et al. 2008], altruistic players, who care about the welfare of their direct neighbors in the social network, are introduced into the inoculation model, which we discussed in detail in Section 4.2.2. The expected social cost in this non-selfish environment is compared to the expected social cost in a purely selfish environment. In the non-selfish environment, the players try to minimize their perceived cost, which is the sum of their actual cost and the actual costs of their neighbors multiplied by a 
friendship factor $F$. Formally, the expected cost of an altruistic player $i$ is

$$
L_{i} f_{i}(\boldsymbol{x})+C_{i} x_{i}+F\left(\sum_{j \in \mathcal{N}_{i}} L_{j} f_{j}(\boldsymbol{x})+C_{j} x_{j}\right),
$$

where $\mathcal{N}_{i}$ denotes the neighbors of player $i$. The friendship factor captures the extent to which players care about their friends, i.e., the players adjacent to them in the social network.

\subsection{Malicious Players}

In most studies, the adversaries are not modeled as strategic players or, equivalently, their strategies are assumed to be exogenously given. In practice, however, the investment decisions made by the players can influence the actions of the adversaries. For example, a rational adversary might opt to focus her resources on attacking players who have invested less and, therefore, are more vulnerable to attacks, which can mean a higher payoff for the adversary. Similarly, using popular software products increases usability, but it also increases the number of attacks due to the attacker optimization strategies mentioned in Section 2.3. The given ecosystem greatly influences the strategic decisions of the attacker in whether she performs generic attacks against a large set of targets or she executes a more targeted operation. Only recently did some researchers [Herley 2010; Laszka et al. 2013] and practitioners [Microsoft 2011] brought this important distinction to the attention of the security community. We believe that this distinction in threat modeling can bring substantial benefit to the community.

In [Hausken 2006], all the adversaries are represented by a single player, called the agent. The model is studied with both exogenous and endogenous adversarial strategies. Endogenous adversarial strategies create negative externalities between players' security investments, which we discussed in Section 4.4.

In [Moscibroda et al. 2006], in addition to the inefficiencies caused by the selfishness of players, some players are allowed to be malicious. As a simplifying assumption, these so called byzantine players have the same set of strategies as the selfish players and cannot be distinguished from them, but their goal is to deteriorate the overall system performance without any regard to their own costs. ${ }^{14}$

\subsection{Risk-Averse Players}

In practice, individuals are generally believed to be risk-averse, which is most commonly modeled using a utility function $u_{i}$, which quantifies the desirability of different outcomes for a given player $i$. If the risk function $f_{i}(\boldsymbol{x})$ measures the probability of a security breach at player $i$, which implies $f_{i} \in[0,1]$, then the expected payoff of player $i$ can be computed as

$$
f_{i}(\boldsymbol{x}) u_{i}\left(W_{i}-L_{i}-C_{i} x_{i}\right)+\left(1-f_{i}(\boldsymbol{x})\right) u_{i}\left(W_{i}-C_{i} x_{i}\right),
$$

where $W_{i}$ is the initial wealth (or endowment) of player $i$ [Ogut et al. 2005; Lelarge and Bolot 2008]. Note that we did not introduce $W_{i}$ in the core model as it does not affect the decisions of risk-neutral players. ${ }^{15}$

The utility function $u_{i}$ is assumed to be monotonically increasing $\left(u_{i}^{\prime}>0\right)$, which implies that outcomes with higher monetary value are more desirable, and concave $\left(u_{i}^{\prime \prime}<0\right)$, which implies risk-aversion due to the diminishing marginal utility. In [Ogut

\footnotetext{
${ }^{14}$ Arguably, there are other attacker threat models. The profit-optimizing attacker model seems to be more realistic in general.

${ }^{15}$ In [Ogut et al. 2005; Lelarge and Bolot 2008], a player also has the option of investing in insurance, which we discuss in Section 7.2.1.
} 
et al. 2005], the model also assumes constant absolute risk aversion (CARA) given by a constant ratio $-\frac{u^{\prime \prime}}{u^{\prime}}$.

\section{EQUILIBRIA AND EFFICIENCY OF INTERDEPENDENT SECURITY GAMES}

Game theory allows us to model the strategic interaction of decision-makers in information security. These games enable us to derive results about the equilibrium information security investment of the population of players. Furthermore, the authors in the literature use existing and novel metrics to characterize the efficiency of the equilibria compared to the achievable total social welfare. In this section, we present equilibrium and efficiency results and discuss the guidelines they present towards improving information security.

We follow the classic methodology of game theory to describe the equilibrium solutions. First, we present existence and computability results on Nash equilibria in interdependent security games in Section 6.1. Then, we discuss the efficiency of these equilibria compared to the social optimum in Section 6.2. Next, in Section 6.3, we present results comparing the efficiency of different equilibria to each other. Section 6.4 considers the effects of incomplete information. Then, we discuss how the game changes in the presence of byzantine players in Section 6.5. Finally, in Section 6.6, we present results on how improvement in security technology affects the players' investment decisions.

\subsection{Existence, Multiplicity, and Computability of Nash Equilibria}

One of the principal questions regarding any game is whether it has an equilibrium solution or not. In the overwhelming majority of the surveyed papers, the equilibrium concept is the Nash equilibrium which is defined as follows.

Definition 6.1 (Nash equilibrium). A set of strategies is a Nash equilibrium if no player can increase her utility by unilaterally deviating from her equilibrium strategy.

In general, such equilibrium exists for interdependent security games. For discrete investment strategies, in [Heal and Kunreuther 2004], it is shown that there always exists a pure-strategy Nash equilibrium in the positive externalities class of problems, which also holds if there are negative externalities. For continuous investment strategies, in [Jiang et al. 2011], it is shown that there always exists some pure-strategy Nash equilibrium in their general model of interdependence. Since these general models, which we introduced in Section 4.1, incorporate most of the other interdependence models as special cases, the results also hold for the majority of the other models.

However, there are some exceptional models to which the above general rule does not apply. For example, if negative externalities dominate, there might not be a purestrategy equilibrium. In the weakest-target model of [Grossklags et al. 2008], the game does not have any pure-strategy equilibrium for non-trivial parameter values; however, a mixed-strategy Nash equilibrium exists. On the other hand, in the weakesttarget model with mitigation of [Grossklags et al. 2008], a pure-strategy equilibrium may exist (besides a mixed-strategy one). As another example, the extended stochastic one-hop model of [Chan et al. 2012] does not have a pure-strategy equilibrium either, due to the negative externalities.

The number of Nash equilibria can also depend on both the model and its parameters. For example, in [Lelarge and Bolot 2008], there is always a unique equilibria in the case of strong protection, but there can be one or two equilibria depending on the parameters in the case of weak protection. The game presented in another work [Miura-Ko et al. 2008b] has a unique NE if the connection/weight matrix of the influence network is strictly diagonally dominant. The number of equilibria can also 
be infinite. For example, in [Omic et al. 2009] it is shown that a SIS epidemic model based game can have an infinite number of equilibria if equilibrium is reached at the threshold of extinguishing the epidemic. As the multiplicity of equilibria can be very important to the efficiency of the system, it is discussed in more detail in the following subsection.

Efficient algorithms for computing a Nash equilibrium have been proposed in several papers. In [Kearns and Ortiz 2004], an algorithm with $O\left(N^{2}\right)$ time complexity is given for computing a pure-strategy Nash equilibrium in their stochastic one-hop propagation based interdependence model. In [Heal and Kunreuther 2004], a polynomial-time algorithm is given for finding a pure-strategy Nash equilibrium in their general, discrete investment strategy based, positive externalities model. The proposed algorithm also works if there are negative externalities. In [Aspnes et al. 2006], it is shown that finding an arbitrary pure-strategy Nash equilibrium in the basic inoculation game is easy: starting from any pure-strategy profile, if at each step some player with a suboptimal strategy changes her strategy, then the profile converges to a Nash equilibrium in at most $2 N$ steps. Consequently, a Nash equilibrium can be computed in $O\left(N^{3}\right)$ time. In [Miura-Ko et al. 2008b], an iterative algorithm, called Asynchronous Best Response Dynamics (ABRD), is proposed to compute the unique pure-strategy Nash equilibrium in the linear influence model. Finally, in [Chan et al. 2012], the authors propose a polynomial-time algorithm for enumerating all mixed-strategy equilibria in their extended stochastic one-hop model, given that the adversary attacks only a single player. Unfortunately, the problem of finding an equilibrium is NP-hard for some games. For example, in [Kumar et al. 2010], it is shown that even determining whether an instance of the generalized inoculation game with hop limit $l$ has a pure-strategy equilibrium is NP-hard, where $1<l<\infty$.

If an equilibrium state is desirable and some of the players are byzantine, then these players may try to prevent the system from reaching an equilibrium or, if the system is already in one, to dislodge it. Preventing the system from reaching an equilibrium forces the honest players to keep changing their strategies continuously, incurring costs and potentially hindering security. In [Moscibroda et al. 2006], the minimum number of byzantine players that can prevent an inoculation game from reaching an equilibrium is studied. A game is called $B$-instable if $B$ byzantine players are sufficient under the assumption that selfish players are not aware of the presence of byzantine players. It is shown that the virus inoculation game is generally 1-instable, but for a certain restricted class of network graphs, it is not 1-instable. Unfortunately, the inoculation game is always 2-instable, which implies that a very low number of attackers masquerading as honest participants can prevent a system from reaching an equilibrium.

\subsection{Efficiency of Nash Equilibria and Free-Riding}

The efficiency of a game's Nash equilibrium solution can be measured against the socially optimal strategy profile. This social optimum is usually defined as the minimum of the sum of the players' individual costs. The metric is relevant because a regulator, also called a social planner, would try to optimize this total social welfare. ${ }^{16}$ The efficiency is typically expressed as the ratio of one of the game's equilibria - usually the pessimistic worst-case equilibrium [Koutsoupias and Papadimitriou 1999] - and the

\footnotetext{
${ }^{16}$ One criticism of social optimum as an optimization goal is that social optima are not necessarily fair, and hence alternative, fairness-respecting metrics should be considered. Let us also mention that in [Omic et al. 2009], the social cost is not computed as the sum of individual costs, but using a "social" unit cost of investment.
} 
social optimum. In this subsection, we discuss some of the most important inefficiency results and the prevalent efficiency metrics.

In many interdependent security game models, efficient equilibria simply cannot exist. For example, in [Kunreuther and Heal 2003], it is shown that for certain parameter values, a stochastic one-hop propagation model can lead to a game that has the same characteristics as the prisoner's dilemma, leading to a single equilibrium in which no player invests in security. In [Varian 2004], it is shown that in the total effort interdependence model, investments levels are always too low in the equilibrium compared with the socially optimal levels. In [Lelarge 2009], it is shown that the equilibria in their epidemic model are always socially inefficient as long as investment externalities are positive. In [Böhme 2012], it is shown that in their stochastic one-hop propagation model, the equilibria are always located below the social optimum if there is any positive degree of interdependence.

In some models, efficient equilibria can exist, but are very volatile. For example, in [Grossklags et al. 2008], it is shown that in the weakest link interdependence model with an insurance option, the equilibria in which players invest a positive amount in security are very volatile when there are many players. That is, the slightest rumor that one player may decrease her investment level is able to make the equilibrium collapse.

Based on these inefficiency results, one might conclude that positive externalities are inherently destructive and the players are always better off if they are independent. However, if the positive externalities are caused by security information sharing, they are usually beneficial. In this case, the social cost in the equilibria of the games is high only when compared to the social optima, but it is low compared to the social cost in the case of independent players. In [Gordon et al. 2003], it is shown that in a general two-player model, the social cost in the equilibrium in the case when there are positive externalities is always less than in the case of independent players. However, if the comparison is based solely on the level of achieved security, positive investment externalities can have a negative effect. In [Gordon et al. 2003], it is shown that even though social welfare is always increased, the overall level of security might be reduced. This can be explained by the positive externalities' mainly negative effect on investment decisions. In [Gordon et al. 2003] and [Ogut et al. 2005], it is shown that in general continuous investment models, the optimal investment level of each player with positive externalities is lower than or equal to the optimal level without externalities.

One of the most widely used metrics for quantifying the inefficiency of a game is the Price of Anarchy (PoA), which was introduced in [Koutsoupias and Papadimitriou 1999]. The Price of Anarchy is the worst-case ratio between the social cost of a Nash equilibrium and the social optimum. This shows how much information security could be improved if appropriate regulations are introduced.

In [Jiang et al. 2011], the Price of Anarchy is analyzed in a general interdependence model with continuous investments. It is shown that, for any given equilibrium $x$, the ratio between the social cost at $\boldsymbol{x}$ and the social optimum, denoted by $\rho(\boldsymbol{x})$, is bounded by

$$
\rho(\boldsymbol{x}) \leq \max \left\{1, \max _{k}\left\{\frac{-\sum_{i} \frac{\partial f_{i}(\boldsymbol{x})}{\partial x_{k}}}{C_{k}}\right\}\right\}
$$


This results is used to analyze two concrete interdependence models, effective investment and bad traffic. In the effective investment model, the PoA is

$$
\text { Po } A \leq \max _{k}\left\{1+\sum_{i: i \neq k} \hat{\omega}_{i k}\right\},
$$

where $\hat{\omega}_{i j}=\frac{C_{i}}{\omega_{i i}} \frac{\omega_{i j}}{C_{j}}$ is the "relative importance" of player $j$ to player $i$. In the bad traffic model, the PoA is

$$
P o A \leq 1+\max _{i, k: i \neq k} \frac{L_{i} \tau_{k i}}{L_{k} \tau_{i k}} .
$$

Note that the bounds are tight in both cases.

In [Aspnes et al. 2006], it is shown that the Price of Anarchy in the basic inoculation game is $\Theta(n)$. In [Kumar et al. 2010], it is shown that when the disease hop limit is $l=1$ and players are uniform in the generalized inoculation game, the Price of Anarchy is at most $\max _{i} d_{i}+1$, where $\max _{i} d_{i}$ is the maximum degree in the player interdependence graph.

One of the main causes for these inefficiencies is the presence of free-riding: interdependent players tend to underinvest and "free-ride" on the positive externalities created by the investments of the other players.

In the general two-player model of [Gordon et al. 2003], it is shown that, at the equilibrium, a small increase in security investments by either player would decrease social cost, which indicates the presence of free-riding. The extent of free-riding can be very extreme in some cases. For example, in the total effort model of [Varian 2004], the level of security is determined by the player with the highest ratio of unit loss to unit cost. Consequently, all other players free-ride on this single player. However, it is also possible that a player invests more in security in an equilibrium than the socially optimal level [Gordon et al. 2003]. In this case, even though the level of security is higher, the player's strategy is economically suboptimal due to the costs of overinvestment in security.

In [Miura-Ko et al. 2008b], a metric, called the Free-riding Ratio, is proposed to quantify the extent of free-riding. Formally, the Free-riding Ratio $\gamma_{i}$ of player $i$ is the ratio of the externalities produced by the neighbors of $i$ over the amount she would invest in isolation. If $\gamma_{i}<0, i$ is forced to over-invest, since the contribution of her neighbors is negative. If $\gamma_{i}=0$, there is no free-riding in either positive or negative sense. If $0<\gamma_{i}<1$, there is limited free-riding, but $i$ still invests a positive amount. Finally, if $\gamma_{i} \geq 1$, there is complete free-riding, which means that $i$ invests nothing and depends completely on her neighbors. The equilibrium values of the free-riding ratios are computed for three example scenarios in [Miura-Ko et al. 2008b], and are used to analyze the scenarios.

When studying the efficiency of a system, it is important to determine how well it "scales", i.e., as the size of the system increases, how much its efficiency decreases. In the case of interdependent security games, we can consider a game to be scalable if it retains its efficiency as the number of players increases.

Unfortunately, related work below shows that most interdependent security games do not scale well. For example, in [Varian 2004], it is shown that in the total effort interdependence model with identical players, the equilibrium investment level remains constant as the number of players increases, but the socially optimal amount of investment increases; thus, the game becomes more inefficient. In [Grossklags et al. 2008], it is shown that in the total effort interdependence model, an equilibrium in which every player invests becomes more and more unlikely as the number of players increases. In 
the stochastic one-hop propagation based model for computer security of [Kunreuther and Heal 2003], it is shown that increasing the number of players increases the negative externality to an investing player if the other players are not investing. Consequently, the incentive for a player to invest diminishes and investing in security can never be a dominant strategy as the number of players grows large. Generally, games based on interdependence models, where the positive effects yielded by the players' investments are shared among every player (e.g., in most linear models), are prone to free-riding if the number of players is high. Similar results exist for propagation based models as well. For example, in [Aspnes et al. 2006], it is shown that the inefficiency (i.e., the PoA) in the basic inoculation game is proportional to the number of players.

There also exist some scalable interdependence games. For example, in [Varian 2004], it is shown that in the weakest link interdependence model with identical players, the socially optimal and the equilibrium risks are identical, regardless of the number of players.

In the case of classic epidemic models, efficiency can be also measured by the equilibrium level of the infection (or by whether the disease extinguishes or not). In [Omic et al. 2009], it is shown that there can be no Nash equilibrium in the SIS model such that the infection rate is below the epidemic threshold, at which the disease extinguishes. In other words, the epidemic is never extinguished by selfish players. In [Theodorakopoulos et al. 2013], a counter-intuitive phenomenon is observed in the SIP model. It is shown that a higher learning rate, which is the rate at which players learn what the infection level is, leads to a higher infection level.

\subsection{Equilibrium Selection}

Besides measuring against an ideal strategy profile, such as a social optimum, the equilibria can also be measured against each other. In the previous subsection, we already discussed the existence of multiple sustainable equilibria in an interdependent security game. If these equilibria have different social costs, a coordination problem arises: the network can be "trapped" in a less desirable equilibrium with a higher social cost, as no individual player has any incentives to change her strategy to the one in the more desirable equilibrium. In this case, there is a possibility of tipping or cascading: inducing a sufficiently large fraction of the players to invest will lead others to follow. Such mechanisms are discussed in Section 7.1.4. Note that in non-deterministic models, such as the stochastic one-hop propagation model, even a single (equilibrium) strategy profile can lead to substantially different outcomes [Laszka et al. 2014; Johnson et al. 2014]. The possibility of multiple outcomes, which can have substantially different social costs, indicates that interdependence can cause systemic risk, a phenomenon which has received only little attention from the research community so far.

In [Grossklags et al. 2010b], the existence of multiple equilibria is listed as one of the key obstacles that may prevent the players from reaching a high security outcome. It is shown that, in both the weakest-link and the total effort interdependence models, there exists a multiplicity of equilibria when security investments and insurance are both available. The existence of less secure and more secure equilibria may cause coordination failures if a single player deviates from investing in security to buying insurance, as a single player deviating might cause other players to follow, which in turn causes the game to end up in a less secure equilibrium.

In [Lelarge and Bolot 2008], the multiplicity of equilibria is studied in the localmean-field epidemic model with weak protection. It is shown that if the cost of protection is in a given range, then everyone and no one investing in security are both Nash equilibria. In this case, the socially optimal strategy profile is always everyone investing. In [Lelarge 2009], it is shown that if the players' potential losses $L_{i}$ are non- 
uniform, there is a possibility for the existence of multiple Nash equilibria in the case of strong protection as well.

In [Kunreuther and Heal 2003], it is shown that for certain parameter values, everyone and no one investing in security can both be equilibria in a stochastic one-hop propagation model. Regulations are proposed to solve the coordination problem arising when none of the players invests because she believes others would not do so.

The problem of multiple equilibria is also studied in [Heal and Kunreuther 2004]. They characterize games in which every player investing in security and none of the players investing are both equilibria by the threshold cost for investing $c_{i}(K)$. It is also shown that if every player investing and no player investing are both equilibria, then the former strategy profile always Pareto dominates the latter.

\subsection{Incomplete Information}

In [Grossklags et al. 2010a], the notion of Price of Uncertainty is introduced to measure the disadvantage of an expert player when it has incomplete information.

Definition 6.2 (Price of Uncertainty). The Price of Uncertainty (PoU) quantifies the maximum discrepancy in the total expected payoff between complete and incomplete information conditions. The metric is defined in three forms:

- Difference: $\operatorname{PoU}_{1}(L, N)=\max _{C, I \in[0, L]}\left\{E P_{\text {complete }}(C, I)-E P_{\text {incomplete }}(C, I)\right\}$,

- Payoff-ratio: $\operatorname{PoU}_{2}(L, N)=\max _{C, I \in[0, L]} \frac{E P_{\text {complete }}(C, I)}{E P_{\text {incomplete }}(C, I)}$,

- Cost-ratio: $\operatorname{PoU}_{2}(L, N)=\min _{C, I \in[0, L]} \frac{E P_{\text {complete }}(C, I)}{E P_{\text {incomplete }}(C, I)}$,

where $E P_{\text {complete }}$ and $E P_{\text {incomplete }}$ denote the expected payoffs under the complete and incomplete information conditions, and $I$ is the unit cost of insurance ${ }^{17}$. Recall that $C$ and $L$ denote the cost of security investment and the magnitude of potential losses when they are uniform over the players. For the difference and payoff-ratio forms, the initial wealth (or endowment) $W$ is set to $L$, while it is set to zero for the cost-ratio form.

The three forms of the metric are analyzed in three games, which are based on the best-shot, weakest-link and total-effort interdependence models.

The observations for the first two forms of the metric are mostly consistent with each other for all three models. Generally, the PoU is high when the number of players is low, but as the number of players increases, the PoU diminishes. In other words, as the number of players increases, the importance of information decreases. This is fortunate, as gathering complete security information gets more difficult (and/or expensive) as the number of players increases. The combination of the difference metric and the weakest-link game is an interesting exception, as the PoU is not affected by the number of players in this case. The main difference between the two forms is that the PoU increases directly with the potential loss for the difference form, while it is independent of the magnitude of the potential losses for the payoff-ratio form. This difference is readily explained by the difference between the two definitions.

The cost-ratio form is the least useful, since the observations based on it are counterintuitive and often contradict those that are based on the other forms. The explanation is that the cost-ratio metric focuses on comparing costs which are insignificantly small, but whose limiting ratio indicates significant discrepancy.

\footnotetext{
${ }^{17}$ Insurance is discussed in Section 7.2.1. Here, it suffices to know that insurance is another investment option that the player has besides security investments to manage risks.
} 
In [Pal and Hui 2011], a comparison based on the players' behavior regarding security investments is made between a less-informed case, where each player knows her own degree (i.e., the number of neighbors she has) and the distributions of her neighbors' degrees, and a more-informed case, where each player also knows her neighbors' actual degrees. In the less-informed case, if we assume that the degrees of neighboring nodes are independent, each player's investment monotonically decreases with increase in her degree in every symmetric equilibria. In the well-informed case, however, if we assume that the degrees of the neighbors of a node are stochastically independent, we only have that there exists at least one symmetric equilibrium in which each player's investment monotonically decreases with increase in her degree. Thus, with increasing information, the increments in overall network security might follow the same trends as in the case when players have less information.

\subsection{Byzantine Players}

The presence of byzantine players can result in an increased social cost due to their malice. In [Moscibroda et al. 2006], the concept of "price of malice" is introduced to measure the excess cost caused by a given number of byzantine players.

Definition 6.3 (Price of Malice). The Price of Malice (PoM) is the ratio between the worst Nash equilibrium with $B$ byzantine players present and the PoA in a purely selfish system. Formally,

$$
\operatorname{PoM}(B)=\frac{\operatorname{PoB}(B)}{\operatorname{PoA}},
$$

where $\operatorname{PoB}(B)$ is the ratio between the worst-case social cost of a NE with $B$ byzantine players divided by the minimal social cost.

The Price of Malice is studied in two models of awareness: oblivious and non-oblivious.

In the oblivious model, the selfish players are not aware of the existence of byzantine players, that is, they assume that all the other players are selfish as well. In this case, players underestimate their probabilities of being compromised and, consequently, the social cost deteriorates as the number of byzantine players increases. Formally,

$$
\operatorname{PoM}(B) \in \begin{cases}\Theta\left(1+\frac{B^{2}}{L}+\frac{B^{3}}{(N-B) L}\right), & \text { when } B<\frac{L}{2}-1, \\ \Theta(L), & \text { otherwise. }\end{cases}
$$

In the non-oblivious model, the selfish players know about the existence and number of byzantine players, but they do not know which players are byzantine. It is also assumed that selfish players are highly "risk-averse": each selfish player presumes that the byzantine players are connected such that her expected cost is maximal. In this case, the players overestimate their probabilities of being compromised and, consequently, are more willing to invest in security. Interestingly, the Price of Malice can be less than 1 in this case, which means that the selfish players' awareness of the existence of byzantine players may lead to an increased investment in security and an improvement in social welfare.

\subsection{Quality of Security Technology}

One might hope that the improvement of security technology, such as the development of better firewalls and intrusion detection systems, will solve the efficiency problems over time. Unfortunately, technology improvement rather has a negative effect on investment decisions.

In [Jiang et al. 2011], it is shown that technology improvement may not offset the negative effect of the lack of incentives, i.e., the PoA does not change with the im- 
provement of security technology, in case of the effective investment and bad traffic interdependence models. Furthermore, if the effectiveness of investments has improved by $a$ times, then the optimal social cost cannot decrease more than $a$ times. In other words, in an interdependent security game, the effect of technology improvement is never amplified, but can rather be diminished.

In [Lelarge and Bolot 2008] and [Lelarge 2009], a similar result is presented for a propagation based local mean field model. It is shown that, for a fixed price, increasing the quality of security technology can lead to a decrease of its adoption.

If the quality and price of security technology is not determined by a competitive market, but by a monopolist provider, the above phenomena has very unpleasant consequences. In [Lelarge 2009], it is shown that a monopolist security provider has no incentives to invest in a high-quality product. If the quality of security is low, the demand is higher because of the positive externalities, of which the monopolist can take advantage. If, however, the quality of security is high, the demand is lower because of the free-rider effect.

\section{IMPROVING SECURITY DECISIONS}

This section draws on the conclusions derived from equilibrium results and surveys related work in which authors proposed game-theoretic solutions and practical mechanisms to improve information security. This improvement does not necessarily mean the increase of the users' security investments, but rather the overall improvement of utilities obtained as a result of better security decisions.

First, in Section 7.1, we consider theoretical results and abstract mechanisms that change the constitution of the strategic situation to set a better equilibrium. Then, in Section 7.2, we discuss practical regulatory and market-based mechanisms for improving the players' security and social welfare.

\subsection{Game-Theoretic Equilibrium Improvements}

In this subsection, we discuss extensions and abstract mechanisms that improve the investments decisions in interdependent security games. These extensions and abstract mechanisms can serve as theoretical bases for designing practical mechanisms for influencing players.

7.1.1. Repeated Game. In repeated games, cooperation is more likely to exist between players. Jiang et al. [Jiang et al. 2011] use the Folk Theorem in repeated games [Fudenberg and Tirole 1991] that proves the support of any feasible and enforceable payoff vector as a subgame-perfect equilibrium (SPE). In their paper, the authors characterize the ratio between the best possible SPE and the social optimum. They found that if individual rationality constraints are effective, then the efficiency of best SPE will be lower than the efficiency of the SO. If these constraints do not hold, then the best SPE can achieve SO.

Repeated games can typically improve the equilibrium solution in a game. Nonetheless, one has to take into account the additional coordination and communication overhead that might prevent the players from achieving the otherwise improved solutions. Taking the cost of communication into account, the beneficial effects of repeated interactions sometime dissipate [Jiang et al. 2011].

7.1.2. Sequential Moves. In some cases, having the players make decisions sequentially instead of simultaneously can also improve the equilibrium. In [Varian 2004], it is shown that for two players in the weakest-link interdependence model, the unique equilibrium in the sequential-move game is the same as the most secure equilibrium of the simultaneous-move game. However, for two players in the total effort and best shot interdependence models, the equilibrium in the sequential-move game is always less 
or equally secure compared to the simultaneous-move game. In this case, the player who moves first is at advantage since there are only two possible outcomes and the first mover can choose the one that she prefers. The highest level of security in the sequential-move game can be achieved by making the player with the lower benefit to cost ratio move first.

7.1.3. Correlated Equilibrium. Correlated equilibrium (CE) is a solution concept which generalizes the notion of NE. Let $\mu$ be a probability distribution over the strategy profiles $x$. First, a mediator selects a strategy profile $x$ with probability $\mu(x)$. Then, she confidentially recommends each player $i$ to invest $x_{i}$. A distribution $\mu$ is a CE iff, for every player $i$, the recommended strategy $x_{i}$ is indeed a best response to the randomized strategies of the other players with distribution $\mu\left(\boldsymbol{x}_{-i} \mid x_{i}\right)$. In other words, it is a NE for all players to follow the recommendation of the mediator.

In practice, the role of the mediator can be played by a trusted third party, such as a government agency. Alternatively, the players can agree on a distribution $\mu$ at a pre-play meeting and later use a device that generates and distributes the appropriate strategies. Furthermore, it was shown in [Stoltz and Lugosi 2007] that CE can arise in an infinite repeated game without a third party or a pre-play meeting. If each player observes the history of the actions of the other players and chooses her action in each period based on a "regret-minimizing" criterion, then the empirical frequencies of the actions converge to a $\mathrm{CE}$.

In [Jiang et al. 2011], the analysis is restricted to CE whose support is on a discrete set of strategy profiles, called discrete $C E$. Both the best and the worst-case discrete $\mathrm{CE}$ are studied. First, it is shown that in a general interdependence model based game, a discrete CE might not achieve the social optimum; however, it can be better than all $\mathrm{NE}$ of the game. Second, it is shown that the PoA of discrete CE is equal to the PoA of pure-strategy NE in the effective investment and bad traffic interdependence models.

In Section 6.5, we discussed the counter-intuitive phenomenon where the presence of malicious players improves social welfare by inducing fear. In [Díaz et al. 2009], the authors study the question whether this "windfall of malice" can be achieved by a mediator without the actual presence of malicious players. It is shown that the mediator can implement a correlated equilibrium by randomly choosing between two types of strategy profiles, an optimal and a "fear inducing" one. In the second one, whose only purpose is to ensure that the selfish players follow the recommendation, any player who does not invest in protection has about $1 / 2$ probability of being infected. It is shown that with such a mediator, the social cost for a regular grid is $\Theta\left(n^{2 / 3} L^{1 / 3}\right)$, which can be a significant improvement compared to the $\Theta(n)$ equilibrium social cost without a mediator.

7.1.4. Tipping and Cascading. If a game has multiple Nash equilibria, it is possible that the players get "stuck" in a less desirable equilibrium. In this case there is a probability of tipping or cascading: inducing some of the players to invest in security will lead others to follow suit.

To study tipping, the concept of critical coalitions is introduced in [Heal and Kunreuther 2004]. If no player investing is an equilibrium, a set of players $\{M\}$ forms a critical coalition if $c_{i}(M) \geq c_{i}, \forall i \notin\{M\}$, i.e., if every other player is better off investing in security given that the members of the critical coalition do invest. It is shown that, if a minimal critical coalition exists, then it has to consist of the players with the highest indirect losses. Furthermore, a minimal critical coalition exists only if the non-additivity $\alpha$ of direct and indirect losses is greater than zero.

In practice, a regulatory authority or an association is more interested in a cheapest critical coalition than a minimal one. If the cost of persuading a single player to invest 


\begin{tabular}{|c|c|c|c|}
\hline Mechanism & $\begin{array}{l}\text { Regulatory / } \\
\text { market-based }\end{array}$ & $\begin{array}{l}\text { Incentive / } \\
\text { dictate }\end{array}$ & Related work \\
\hline insurance & $\begin{array}{l}\text { both (e.g., } \\
\text { mandatory } \\
\text { insurance) }\end{array}$ & incentive & $\begin{array}{l}\text { [Kunreuther and Heal 2003] } \\
\text { [Ogut et al. 2005] } \\
\text { [Grossklags et al. 2008] } \\
\text { [Grossklags et al. 2010a] } \\
\text { [Pal and Hui 2011] }\end{array}$ \\
\hline bonuses \& penalties & regulatory & incentive & $\begin{array}{l}\text { [Gordon et al. 2003] } \\
\text { [Varian 2004] } \\
\text { [Grossklags et al. 2010b] }\end{array}$ \\
\hline liability & regulatory & incentive & $\begin{array}{l}\text { [Kunreuther and Heal 2003] } \\
\text { [Varian 2004] } \\
\text { [Ogut et al. 2005] }\end{array}$ \\
\hline subsidies \& fines & regulatory & incentive & $\begin{array}{l}\text { [Kunreuther and Heal 2003] } \\
\text { [Heal and Kunreuther 2004] } \\
\text { [Omic et al. 2009] } \\
\text { [Grossklags et al. 2010b] } \\
\text { [Amin et al. 2011] }\end{array}$ \\
\hline regulations & regulatory & dictate & $\begin{array}{l}\text { [Kunreuther and Heal 2003] } \\
\text { [Grossklags et al. 2008] } \\
\text { [Omic et al. 2009] }\end{array}$ \\
\hline $\begin{array}{l}\text { audits \& third-party } \\
\text { inspections }\end{array}$ & market-based & dictate & [Böhme 2012] \\
\hline coordination & both & dictate & $\begin{array}{l}\text { [Kunreuther and Heal 2003] } \\
\text { [Saad et al. 2010] }\end{array}$ \\
\hline security information sharing & regulatory & dictate & $\begin{array}{l}\text { [Gordon et al. 2003] } \\
\text { [Ogut et al. 2005] }\end{array}$ \\
\hline
\end{tabular}

in security when no other player does so is assumed to be equal to the cost of the security investment, it can be shown that any cheapest critical coalition is also a minimal critical coalition. Consequently, in general, the unique minimal critical coalition of a game is also its unique cheapest critical coalition.

\subsection{Mechanisms for Improved Security}

In this subsection, we discuss practical mechanisms for improving the level of security and social welfare in interdependent security games. A brief comparison of these mechanisms is given in Table IV.

Please note that the terminology for bonuses/penalties, liability and subsidies/fines varies in the literature. In this survey, bonuses/penalties are rewards/punishments for the security outcome of a player (e.g., a player has to pay a penalty if her security is breached); subsidies/fines are rewards/punishments for the behavior of a player (e.g., a player has to pay a fine if she does not invest in security); and liabilities are special penalties that are equal to the damages caused by the player and are paid to the player who sustained the damage.

7.2.1. Insurance. To date, insurance is probably the most studied remedy to information security investment issues. Cyber-insurance, as it is called in the information security context reduces the chances of a critical loss by distributing the risk among the players. Insurance requires the categorization of players, effectively introducing audit mechanisms. Security audits required by insurance policies subsequently force the participants to maintain a pre-defined level of system security hence improving overall information security. The major issues with insurance are the adverse effects due to externalities (Section 2.1), the large-scale correlation of security incidents due to monopoly markets (Section 2.3), insurance policy enforcement due to information 
asymmetries (Section 2.2), and the lack of available data. The related work on cyberinsurance is extensive, for a comprehensive overview of the papers, we refer to [Böhme and Schwartz 2010]. We will now summarize the issues due to interdependence in these papers.

Information asymmetries between the insurers and the players can have adverse effects on investment decisions, which lead to decreased levels of security and, possibly, decreased social welfare. Insurance discourages investment in security if insurers are unable to detect the careless behavior of the insured players, who know that they will receive compensation should they suffer loss [Kunreuther and Heal 2003]. Consequently, a high security equilibrium may be lost as the players invest in insurance instead of security.

On the other hand, if these information asymmetry problems are eliminated, insurance with actuarially fair premiums encourages a risk-averse player to invest in security whenever the increase in security costs is less than the reduction in expected losses [Kunreuther and Heal 2003]. If insurance is mandatory for the players, security is increased because the players invest more into security as a rational response to the reduction in insurance premiums. Insurance leads to a market solution that is aligned with the economic incentives of both the insurers, who earn profit from appropriately pricing premiums, and the players, who can hedge potential losses [Pal and Hui 2011].

In the case of voluntary insurance, the players' insurance coverage decisions can also be studied. In [Ogut et al. 2005], insurance decisions are assumed to be continuous. As expected, both a higher amount of risk (i.e., expected loss) and a higher degree of risk aversion cause increased insurance coverage. If the level of interdependence is higher, then insurance coverage is less or equal (equality holds when the insurance market is mature). This phenomena might seem counter-intuitive at first because an increased risk (caused by interdependence) should motivate players to take more insurance. However, since the total risk is higher from the insurer's perspective, so is the price of insurance, which counters the increased demand for insurance.

When studying the impact of insurance on interdependent security games, the supply side of insurance also has to be taken into consideration. From the players' perspective, the different characteristics of the supply side can be summarized as the maturity of the insurance market. The maturity of the market is low if

- there are few insurers, and hence little competition,

- adequate actuarial data is unavailable, or

- there exists a high correlation between players' loss events that can cause significant system-wide losses [Ogut et al. 2005].

The price of insurance is determined by the maturity of the insurance market and the level of risk. If the insurance market is mature, the insurers do not make any profit, i.e., the insurance premium paid by a given player is equal to her risk. Immature markets can be modeled through a loading factor, which measures the excess of the premium relative to the risk [Ogut et al. 2005].

In [Ogut et al. 2005], it is shown that insurance market maturity can affect both the insurance and the security investment decisions of the players. As the market becomes more mature, security investments decrease, which can be easily explained by the fact that security investment is more effective than insurance when the insurance market is immature.

The immaturity of the market is obviously disadvantageous for the players due to the increased costs of insurance. However, an immature market can also have some positive effects. For example, a single monopolist insurer can be advantageous because she wants to internalize the externalities [Kunreuther and Heal 2003]. In a 
competitive market, an insurer would be reluctant to reduce the premium of a player for investing in security since she cannot observe or control the investments of the other players, who could cause indirect loss to the client. A single insurer, on the other hand, can require all players to invest in return for premium reductions and, consequently, increasing the overall level of security. As an other example, in [Grossklags et al. 2010a], it is shown that the Price of Uncertainty in the weakest-link interdependence model is the highest when insurance is competitively-priced.

The amount of loss in case of a security breach can also be reduced by using selfinsurance technologies or practices, such as backup provisions [Grossklags et al. 2008; Grossklags et al. 2010a]. Self-insurance can be modeled in the same way as voluntary insurance provided by an insurer with a fixed (unit) price of insurance, which is determined by the employed technology or practice.

7.2.2. Bonuses and Penalties. In [Grossklags et al. 2010b], rebates and penalties are proposed as mechanisms that can be used to shape the incentives of players. A player is subjected to a penalty when her security is broken, and receives a bonus when she remains secure. In [Grossklags et al. 2010b], these mechanisms are proposed as economically-motivated strategies that an ISP may use to influence its customer. In this example, penalties can be implemented as reductions in network throughput or as a quarantine, while bonuses as monetary benefits or reduced subscription costs. Numerical sensitivity analysis shows that in general, bonuses and penalties can be more effective than fines and subsidies, which are discussed in Section 7.2.4, for the weakest-link interdependence model [Grossklags et al. 2010b]. For the total effort interdependence model, it is observed that moderately sized interventions have little impact, which can be explained by the rapid decrease in the incentive for investing in security as the network grows in size. Consequently, penalties need to be in proportion with the size of the network to have a noticeable impact. It is also noted that such a policy needs to be well-balanced as most users disfavor penalty-based systems.

In [Varian 2004], the optimal penalty, which induces socially optimal levels of investment, is studied. It is shown that the penalty should be imposed on the player who has the lowest cost of reducing the probability of security breach and that the penalty should be equal to the losses of the other players. It is noted that the principle of the liability of the player with the least cost is a standard result in the economic analysis of tort law, where it is sometimes called the doctrine of the "least-cost avoider".

In [Gordon et al. 2003], a special penalty rule is proposed. Under this rule, if a player causes damage to other players, then she is charged the value of the difference between the realized losses of the other players and their expected losses at the social optimum. It is shown that this mechanism fully internalizes externalities and makes each player's objective of minimizing her own expected cost equivalent to minimizing the social cost function up to a constant.

7.2.3. Liability. A very straightforward way of internalizing externalities is to hold players liable for the damages they cause to other players because of their negligence. Liability can be thought of as a special penalty, whose value is equal to the amount of damages caused and which is paid to the players who sustained the damage.

In [Ogut et al. 2005], the liability system is mathematically analyzed and it is shown that when players maximize their individual utility, security investment levels are higher with liability than without.

Unfortunately, the liability system can not be considered a perfect solution for multiple reasons. In [Kunreuther and Heal 2003], it is observed that the liability system, despite having attractive theoretical properties, faces practical problems due to high transaction costs, since determining the cause of a loss can be very costly (think about the cost of a forensics investigation involving security experts). Furthermore, in [Ogut 
et al. 2005], it is shown that security investment levels with liability are higher than the social optimum level without liability. That is, liability can make players overinvest in security compared to the socially optimal level. Finally, in [Varian 2004], it is shown that in the total effort model, if the liability payment is too large, it may induce a player to seek to be damaged.

In [Varian 2004], it is also shown that liability is not adequate in general to achieve socially optimal levels of investment in the weakest link model. In such cases, a negligence rule can be used to induce optimal investments. Under the doctrine of the negligence rule, a regulatory authority determines the level of due care prior to the game. Then, in the event of a security breach, a player can be held liable only if her investment level is below the level of due care. It can be shown that the negligence rule induces optimal investment decisions in the weakest link and many other similar models, such as the total effort model [Varian 2004]. It is noted that this is a standard result in liability law.

7.2.4. Subsidies and Fines. Subsidies/fines might seem to be similar to bonuses/penalties at first sight, but there is a fundamental difference between the two mechanisms: the former rewards/punishes the effort of a player, while the latter rewards/punishes the outcome [Grossklags et al. 2010b].

In [Kunreuther and Heal 2003], it is proposed that the public sector could intervene directly in free-riding problems by levying a fine on players who do not invest in security or, equivalently, by providing a subsidy to players who do invest.

In [Amin et al. 2011], a fine is suggested to alter the individually optimal security choices, in which the players tend to under-invest in security relative to the socially optimal choices. It is shown that a range of penalties can be computed such that the individually optimal choices in the game with penalties coincide with the socially optimal ones.

In [Omic et al. 2009], it is shown the Nash equilibrium of the virus protection game depends on the vector of the unit costs of investment $C$. By varying $C$, a "network manager" (e.g., the public sector) can influence the network equilibrium point. One way of adjusting the unit cost is through subsidizing the cost of security investments (e.g., the price of antivirus software); for example, players who have many interactions and are densely connected can be given cheaper (per unit) antivirus. Another possible way of adjusting the relative cost of insurance is to levy a fine on those players who do not invest. Some conditions are introduced in [Omic et al. 2009] that can give guidance to choosing the right values for the costs of security investments. If all $C_{i}>1$, there is only one equilibrium, in which no player invests in security. If $C_{i}<\frac{1}{d_{i}}$, a player always invests a positive amount in security. Finally, too low relative prices can lead a network further away from the optimum: if a densely connected player invests in expensive security, other players can invest less such that the network reaches the epidemic threshold.

In [Grossklags et al. 2010b], subsidies are discussed as a mechanism that ISPs can use to influence customer behavior. For example, security products can be offered at a reduced cost. Similarly to bonuses and penalties, it is observed that subsidies and fines only work at margin in the total effort interdependence model, when the subsidizer provides security products free of charge [Grossklags et al. 2010b].

7.2.5. Regulations. Instead of relying on economic incentives, such as subsidies or liabilities, to influence the investment decisions of the players, a social planner might be able to dictate decisions using regulations. 
In [Kunreuther and Heal 2003], the question under what conditions should regulations be considered is studied. In an example of $N$ identical players, regulations are shown to be desirable from both private and social welfare perspectives if

— there are two stable Nash equilibria, in which everyone and no one invests in security,

- the equilibrium where everyone invests yields higher payoffs for all players than the equilibrium where no one invests, and

- none of the players voluntarily invested in security because they believed others would not do so.

Therefore, regulations should be considered when the cost of security investment is between the threshold under which investing is always optimal (regardless of the decisions of the other players) and the expected loss through direct risk. In this case, regulations solve a coordination problem.

In [Omic et al. 2009], imposing upper bounds $\Gamma_{i}$, for $i=1, \ldots, N$, on the infection probabilities in the virus protection game is studied. These bounds can serve as a form of strict regulation, which requires the players to reach a level security, regardless of the costs incurred. Two particular upper bound settings are discussed.

- If $\Gamma_{i} \rightarrow 0$ for a given player $i$ and $\Gamma_{j}$ is finite for every other player, the curing rate of $i$ (i.e., the investment of $i$ ) will tend to infinity.

- If $\Gamma_{j}=\Gamma$, there exists a feasible strategy profile in which every player invests an amount that is proportional to her degree in the network $d_{i}$. Unfortunately, this is not a stable point: if there is an unfair player, who reduces her investment against the rule such that her infection probability rises above the bound, she can cause the other players to invest more than what was planned.

The latter result suggests a strategy for steering autonomous systems (ASs) to invest an amount in security that is proportional to the amount of interactions they have with other ASs [Omic et al. 2009]. Security can be enforced by requiring their infections probabilities to be under a certain fixed bound. Together with the fact that the cheapest threshold, in terms of total security investment, is reached when the players invest proportionally to their own degrees, this is a very fair way to provide overall security.

If negative externalities dominate, such as in the weakest target model of [Grossklags et al. 2008], the social planner has to either create a "honeypot player" or, if that is not an option, to select an individual to act as a target. Unfortunately, if insurance is not available or too expensive, the selected player essentially sacrifices herself. The willingness of individuals to serve as "sacrificial lambs" has been studied by anthropology and economics [Grossklags et al. 2008].

Regulations are only useful if they can be enforced. In order to do that, one has to first reliably measure the security level of players and their investments. In practice, security audits and third-party inspections, which are discussed in the following subsection, are commonly used for this.

One way for the public sector to enforce regulations is to turn to the private sector for assistance [Kunreuther and Heal 2003]: third-party inspections coupled with insurance protection can encourage players to reduce their risks from incidents. Such a management-based regulatory strategy forces the players to do their own planning as to how they meet the regulations, instead of regulatory decision-making. 
7.2.6. Audits and Third-Party Inspections. Regulations prescribe rules for the players, but additional mechanisms are needed to enforce these rules ${ }^{18}$. Audits and third-party inspections are required to check the compliance of the players to the regulation. Security audits can generate positive utility through two channels [Böhme 2012]:

- First, they can help overcoming information asymmetries described in Section 2.2. Security products constitute a lemon market, which results in the price for goods of unknown quality dropping to the price of insecure goods. Audits can be used to signal the quality of security and, thus, establish a market for secure products.

- Second, they can solve coordination problems. Audits can be used as credible signals, which the players can use to announce information about their investment and security levels. This allows new, socially better equilibria that would not be stable otherwise.

Of these two channels, the first one affects the relationship between a player and an external entity; hence, it is not directly connected to interdependent security games. The second one, on the other hand, can be used to improve the interdependent players' security decisions through coordination.

In [Böhme 2012], the author studies the question under which conditions do security audits generate positive utility by solving the coordination problems, which would otherwise hinder the reduction of interdependent risks. Based on the degree of interdependence and the security productivity ${ }^{19}$, the following equilibrium situations are identified:

- If the degree of interdependence is low, players always have incentives to invest at or above a certain level. Therefore, audits below this level are ineffective. Thorough audits, however, can improve social welfare. Since this involves coordination at nonequilibrium points, such audits have to be bilateral.

- If the degree of interdependence and the level of security productivity are both high, there exists three Nash equilibria. In one of them, all players abstain from investment. In this case, security audits can be maximally effective in solving the coordination problem between multiple equilibria. Unilateral audits above a certain level are enough to move all players to the best possible equilibrium. However, even the best possible equilibrium is below the social optimum. To further approach the optimum, more through, bilateral audits are needed.

- If the degree of interdependence is high, but the level of security productivity is low, there exists exactly one Nash equilibrium, in which all players abstain from investment. This case is not a coordination game in the strict sense; therefore, the effectiveness of all audits is limited. Audits may contribute to higher security level if all players perform bilateral audits. Unilateral audits are less effective in general and completely ineffective for a certain range of the parameters.

- If the degree of interdependence is very high and the level of security productivity is very low, there exists exactly one Nash equilibrium, in which all players abstain from investment, which concurs with the corner solution of the social optimum. In this case all audits are useless. Mandatory audits with sanctions would induce overinvestment and decrease social welfare.

- If the degree of interdependence is zero (i.e., there is no interdependence at all), there exists exactly one Nash equilibrium which concurs with the social optimum.

\footnotetext{
${ }^{18}$ Another way to improve security is to establish industry good practices, but they typically remain recommendations only with no enforcement power.

${ }^{19}$ For the definitions of these parameters, see Section 4.3.
} 
One of the main implications of the analysis is that effectiveness is very sensitive to the situation as unfitting audits are often useless. As a solution, audits should best be designed in a modular manner to allow tailored examinations. However, the first situation can serve as a rule of thumb, since it covers more than half of the parameter space: audits at very low security levels are often ineffective; therefore, they should be focused on the possibility to extract verifiable information about high security levels. Finally, mandatory audits seem unnecessary in situations where the players have their own incentives to conduct audits.

7.2.7. Coordination and Cooperation. In the absence of a social planner, the players can choose to cooperate for the common goal of reducing social cost and coordinate the game themselves.

In [Kunreuther and Heal 2003], two non-centralized coordinating mechanisms are discussed, both in the context of airline security. First, an association of players could play a coordinating role by requiring every member to follow certain rules and regulations, including the adoption of security measures. The association could then refuse to do business with players who are not members and/or not follow the rules. Second, players who have invested in security could announce publicly that they will not do business with players who have not done so. This tactic may encourage irresponsible players to invest in security.

In [Saad et al. 2010], coalitional game theory is used study the cooperation between players whose security is interdependent. The players can form cooperative groups, i.e., coalitions, which allow them to

- improve the positive effects of their security investments and

- reduce the negative effects of their threats on the other players of the same coalition.

The formation of coalitions also entails costs for the players. First, there are usually natural frictions between the players due to differences that need to be overcome, which can be modeled by a friction matrix, where each element is the degree of friction between a pair of players. Second, coordinating the coalition requires effort from the participating players, which can be modeled by a cost that is proportional to the size of the coalition. The model is used to establish the necessary and sufficient conditions under which it is beneficial for two coalitions to merge into one. These results are applied in the study of an example network, which models the cooperation between the different divisions of a large company that offer video-on-demand services.

7.2.8. Sharing of Security Information. In [Gordon et al. 2003], security-based information sharing organizations (SB/ISOs) are studied in a general two-player model. In this model, if player $i$ shares security information with the other player, a portion (denoted by $\theta_{i} \in[0,1]$ ) of her security investment benefits the other player without diminishing the benefit of the providing player. It is shown that information sharing always decreases the social cost through increased positive externalities. Consequently, if there are no enforcement costs associated with a sharing policy, the mandated degree of sharing should always be increased. It is also shown that without mandatory sharing, players have no incentives to share security-based information: if the players are free to select their sharing portions, the only equilibrium is when the portions of both players are zero. This discrepancy between the socially and individually optimal strategies (i.e., between sharing and not sharing) implies that there is a greater need for cooperation when information sharing is possible.

In [Ogut et al. 2005], two models of information sharing are analyzed. First, information sharing reduces direct attack probability, but not the degree of interdependence. Second, information sharing reduces the degree of interdependence, but not direct at- 
tack probability. In the second case, a central agency informs firms on how to protect themselves from indirect attacks.

\section{SUMMARY AND FUTURE DIRECTIONS}

In this paper, we survey the state-of-the-art of interdependent security games. We also distill the most important core modeling decisions and provide an overview of extensions found in the literature. The game-theoretic models in this survey identify a few key problems in information security investments and the authors propose potential remedies to mitigate these problems. Yet, we believe that several open problems remain that need more attention from the research community. We now present a few of these open problems in the hope of bootstrapping new exciting research in the area.

\subsection{Security Investments}

In interdependent security games, the security investment of the players is modeled either as a discrete or a continuous variable. To keep the models tractable, the discrete security investment is usually defined as a binary decision between full protection or no protection at all. Similarly, continuous investments are easy to use in modeling. This simplifications do not capture the real nature of security modeling, where investment typically happens in discrete steps (such as buying a set of security products or conducting $\mathrm{X}$ number of system tests). Multidimensional security investments are not thoroughly considered in the literature. A player can invest in different types of security mitigating options, for example allocate some budget on user education and/or security technology improvements and/or cyber-insurance. The modeling of this diversity of security options is a potential improvement to many of the existing game-theoretic models.

\subsection{Strategic Adversaries}

Most papers in the interdependent security literature consider the attackers as an exogenous, persistent threat and not as players in a game. Note that the interdependent security models are fundamentally different from research modeling the attackersdefenders interaction as a two-player game. ${ }^{20}$ Nonetheless, the attacker have their strategic incentives and they are working towards maximizing their, mostly unknown, utilities. Moreover, there is evidence that the attacks experienced by the defenders are the result of the cooperation of various participants in the underground economy [Levchenko et al. 2011]. We believe that the proper modeling of strategic adversaries in interdependent security games is a largely undiscovered research area. It was partially untouched, because the utilities of attackers are difficult to judge and quantify. With an increasing number of papers including measurements about the activity underground black markets [Holz et al. 2009], the opportunity opens to develop appropriate game models.

\subsection{Negative Externalities}

In this survey, we have seen that the security investment decisions of players create both positive and negative externalities. Most of the interdependence models focus on positive externalities as they typically rely on relationship information that is easy to model, maybe even known to the players. On the contrary, negative externalities typically arise when attackers substitute a target for another one upon discovering the adequate protection of their original target. The selection involves the rational (or

\footnotetext{
${ }^{20}$ Most papers that do model strategic adversaries consider them in an attacker-defender two-player game. One of the few exceptions including interdependency of the defenders is [Hausken 2006] covered in Section 5.3.
} 
not so rational) decision-making process of the attacker that is notoriously difficult to model. In [Herley 2010], the author point out that there is a scalability issue when modeling attackers, and indeed attackers cannot just target the total population of potential victims. The author argues that finding the right target (i.e., correctly assessing the security posture of the targets) is a key task the attackers need to do and is a modeling aspect most existing models neglect.

\subsection{Topology and Network Modeling}

Most interdependent security models abstract away the real topology of computer networks to be able to formulate closed-form equilibrium and efficiency results. Yet, network topology plays an important role as it is the true basis for security interdependence. Epidemic models come closest to considering the network topology when they model the explicit spreading behavior of a virus and other malware in a network. Nonetheless, epidemic models carry their legacy from biology and thus their assumptions are often inappropriate in computer networks. For example, recovery and resistance in epidemic models do not correspond to the recovery and forensics of computer networks. To date, there is a lack of reliable, extensive and diverse data sources that would enable researchers to verify the predictions of their models in a real-world environment. Very recently, there has been some effort in industry to collect and share extensive security data on a large-scale and make it available to researchers [Dumitras and Shou 2011]. Such datasets will lead to a new avenue of research that hopefully results in more applicable, realistic models and enable the establishment of various security metrics that can be used in risk modeling.

Understanding the impact of network topologies is not the last step. Network topologies emerge from the strategic interaction of players in a global interaction game. One can argue that topology formation is not driven by security concerns, but by other utility components. Yet, we believe that security should be considered when making decisions about whom to connect with, as the resulting topology can have an impact on the emerging security risks. To the best of our knowledge, strategic and secure network formation has not been addressed in the research literature of interdependent security games. We argue that this fundamental emerging property, which not only affects the risks of individual players (individual point of view) but also defines network robustness (social point of view), should be studied in more detail. Understanding the characteristics of strategic network formation should ideally lead to efficient and secure network topologies, otherwise more attention needs to be paid to incentive mechanisms to drive the players towards robust and secure networks.

\subsection{Reducing Uncertainty and Information Sharing}

One of the key factors to hamper proper security investments is the inability of players to assess their environment, the risks they face and the cost of the potential options to mitigate these risks. We touch upon a few papers in this survey that address uncertainty in security investment decision-making. We believe that the lack of transparency in security is a significant problem that reinforces the attackers' advantages. The uncertainties surrounding risks and the benefit from implementing securityimproving remedies can be greatly reduced by establishing extensive, industry-wide datasets for specific domains of security research. The availability of real-world dataset should allow researchers and practitioners to establish widely-accepted risk metrics and security benchmarks. In addition, uncertainty can be greatly reduced across players using information sharing. In practice, industry has established common standard for security information sharing, for example by means of IP blacklists [Sinha et al. 2008]. The authors of [Gordon et al. 2003] show that information sharing reduces the need for security investment for firms while increasing the social welfare (that is they 
are protected with less investments). Yet, the same authors also prove that information sharing is not in the best interest of rational players and if they are to select the amount of information shared, they will select none. Thus external enforcement mechanisms are needed to improve social welfare. Indeed, in practice, information sharing remains a key ingredient of agile, reactive defense solutions, but there is a lot of room for improvement, for example in forensics [Bencsáth et al. 2012] and coordinated action against the attackers' infrastructure in phishing [Moore and Clayton 2008].

\subsection{Dynamic and Repeated Games}

Establishing and maintaining information security is not a static process. Nonetheless, most of the research papers consider single stage (that is one-shot) games. We mention in Section 7.1.1 that repeated games allow players to establish more efficient equilibria. The number of equilibria typically increases in repeated games, but the multitude of equilibria emphasizes the question of equilibrium selection. Equilibrium selection comes with the price of increasing coordination and communication overhead between the players. In an extreme case, the cost of coordination can completely cancel out the benefits of repeated interactions. Thus, the players have to weigh carefully if and how much they are willing to coordinate in order to achieve a better equilibrium in interdependent security games. Since security is inherently a cat-and-mouse game between attackers and defenders, dynamic games seem to be a logical next step as modeling tools. We encourage more research contributions modeling information security using both dynamic repeated and evolutionary games.

\section{REFERENCES}

G.A. Akerlof. 1970. The market for "lemons": Quality uncertainty and the market mechanism. The Quarterly Journal of Economics 84, 3 (Aug 1970), 488-500.

S. Amin, G. A. Schwartz, and S.S. Sastry. 2011. On the interdependence of reliability and security in Networked Control Systems. In Proceedings of the 50th IEEE Conference on Decision and Control and European Control Conference (CDC-ECC 2011). 4078-4083.

Saurabh Amin, Galina A Schwartz, and S Shankar Sastry. 2012. Security of interdependent and identical Networked Control Systems. Automatica 42, 1 (2012), 186-192.

R. Anderson. 2001. Why information security is hard - an economic perspective. In Proceedings of the 17th Annual Computer Security Applications Conference (ACSAC 2001). IEEE, 358-365.

R. Anderson and T. Moore. 2006. The economics of information security. Science 314, 5799 (Oct. 2006), 610613.

J. Aspnes, K. Chang, and A. Yampolskiy. 2004. Inoculation strategies for victims of viruses and the sum-ofsquares partition problem. Technical Report YALEU/DCS/TR-1295. Yale University.

J. Aspnes, K. Chang, and A. Yampolskiy. 2006. Inoculation strategies for victims of viruses and the sum-ofsquares partition problem. J. Comput. System Sci. 72, 6 (September 2006), 1077-1093.

Moshe Babaioff, Robert Kleinberg, and Christos H Papadimitriou. 2007. Congestion games with malicious players. In Proceedings of the 8th ACM Conference on Electronic Commerce (EC 2007). 103-112.

Boldizsár Bencsáth, Gábor Pék, Levente Buttyán, and Mark Felegyhazi. 2012. Duqu: Analysis, Detection, and Lessons Learned. In Proceedings of the 5th European Workshop on System Security (EuroSec 2012). ACM.

R. Böhme. 2012. Security Audits Revisited. In Proceedings of the 16th International Conference on Financial Cryptography and Data Security (FC 2012). Springer, 129-147.

Rainer Böhme and Gaurav Kataria. 2006. Models and Measures for Correlation in Cyber-Insurance. In 5th Workshop on the Economics of Information Security (WEIS 2006).

R. Böhme and G. Schwartz. 2010. Modeling cyber-insurance: Towards a unifying framework. In 9th Workshop on the Economics of Information Security (WEIS 2010).

Michael Ceyko, Hau Chan, and Luis E Ortiz. 2011. Interdependent Defense Games: Modeling Interdependent Security under Deliberate Attacks (Extended Abstract). In Proceedings of the International Conference on Game Theory, 22nd Stony Brook Game Theory Festival of the Game Theory Society. 
Hau Chan, Michael Ceyko, and Luis E Ortiz. 2012. Interdependent Defense Games: Modeling Interdependent Security under Deliberate Attacks. In Proceedings of the 28th Conference on Uncertainty in Artificial Intelligence (UAI 2012).

Defence Signals Directorate. 2012. Top 35 Mitigation Strategies. http://www.dsd.gov. au/infosec/top35mitigationstrategies .htm. (2012). retrieved on Nov 14, 2012.

Josep Díaz, Dieter Mitsche, Navin Rustagi, and Jared Saia. 2009. On the Power of Mediators. In Proceedings of the 5th International Workshop on Internet and Network Economics (WINE 2009). Springer, 455-462. DOI : http://dx.doi.org/10.1007/978-3-642-10841-9_42

T. Dumitras and D. Shou. 2011. Toward a standard benchmark for computer security research: The worldwide intelligence network environment (WINE). In Proceedings of the 1st Workshop on Building Analysis Datasets and Gathering Experience Returns for Security (BADGERS 2011). 89-96.

Drew Fudenberg and Jean Tirole. 1991. Game Theory. MIT Press, Cambridge, MA.

J. Gans, S. King, and G. Mankiw. 2011. Principles of microeconomics. Cengage Learning, Australia.

Lawrence A. Gordon and Martin P. Loeb. 2002. The economics of information security investment. ACM Transactions on Information and System Security 5, 4 (November 2002), 438-457. DOI : http://dx.doi.org/10.1145/581271.581274

L. A. Gordon, M. P. Loeb, and W. Lucyshyn. 2003. Sharing information on computer systems security: An economic analysis. Journal of Accounting and Public Policy 22, 6 (2003), 461-485.

J. Grossklags, N. Christin, and J. Chuang. 2008. Secure or insure? A game-theoretic analysis of information security games. In Proceedings of the 17th International Conference on World Wide Web (WWW 2008). ACM, 209-218.

J. Grossklags, B. Johnson, and N. Christin. 2010a. The price of uncertainty in security games. In Economics of Information Security and Privacy, T. Moore, D. Pym, and C. Ioannidis (Eds.). Springer, 9-32.

J. Grossklags, S. Radosavac, A. Cárdenas, and J. Chuang. 2010b. Nudge: Intermediaries' role in interdependent network security. Proceedings of the 3rd International Conference on Trust and Trustworthy Computing (TRUST 2010) (2010), 323-336.

K. Hausken. 2006. Income, interdependence, and substitution effects affecting incentives for security investment. Journal of Accounting and Public Policy 25, 6 (2006), 629-665.

G. Heal and H. Kunreuther. 2004. Interdependent security: A general model. Technical Report NBER Working Paper No. 10706. National Bureau of Economic Research.

G. Heal and H. Kunreuther. 2005. IDS models of airline security. Journal of Conflict Resolution 49, 2 (2005), 201-217.

C. Herley. 2010. The plight of the targeted attacker in a world of scale. In 9th Workshop on the Economics of Information Security (WEIS 2010).

C. Herley and D. Florêncio. 2009. A profitless endeavor: Phishing as tragedy of the commons. In Proceedings of the 2008 Workshop on New Security Paradigms (NSPW 2008). ACM, 59-70.

C. Herley and D. Florêncio. 2010. Nobody sells gold for the price of silver: Dishonesty, uncertainty and the underground economy. Springer, 33-53.

T. Holz, M. Engelberth, and F. Freiling. 2009. Learning more about the underground economy: A case-study of keyloggers and dropzones. Proceedings of the 14th European Symposium on Research in Computer Security (ESORICS 2009) (2009), 1-18.

L. Jiang, V. Anantharam, and J. Walrand. 2011. How Bad Are Selfish Investments in Network Security? IEEE/ACM Transactions on Networking 19, 2 (April 2011), 549-560. DOI : http://dx.doi.org/10.1109/TNET.2010.2071397

Benjamin Johnson, Aron Laszka, and Jens Grossklags. 2014. How Many Down? Toward Understanding Systematic Risk in Networks. In Proceedings of the 9th ACM Symposium on Information, Computer and Communications Security (ASIACCS 2014).

Michael J. Kearns and Luis E. Ortiz. 2004. Algorithms for Interdependent Security Games. In Advances in Neural Information Processing Systems. MIT Press.

E. Koutsoupias and C. Papadimitriou. 1999. Worst-case equilibria. In Proceedings of the 16th Annual Symposium on Theoretical Aspects of Computer Science (STACS 1999). Springer, 404-413.

P. Krugman, R. Wells, and E. Kelly. 2008. Study Guide for Microeconomics. Worth Publishers. http://books. google.hu/books?id=LP6UCRx5OwkC

VS Kumar, R. Rajaraman, Z. Sun, and R. Sundaram. 2010. Existence Theorems and Approximation Algorithms for Generalized Network Security Games. In Proceedings of the 30th International Conference on Distributed Computing Systems (ICDCS 2010). IEEE, 348-357.

H. Kunreuther and G. Heal. 2003. Interdependent security. Journal of Risk and Uncertainty 26, 2 (2003), 231-249. 
Aron Laszka, Benjamin Johnson, and Jens Grossklags. 2013. Mitigating Covert Compromises: A GameTheoretic Model of Targeted and Non-Targeted Covert Attacks. In Proceedings of the 9th Conference on Web and Internet Economics (WINE 2013). Springer, 319-332.

Aron Laszka, Benjamin Johnson, Jens Grossklags, and Mark Felegyhazi. 2014. Estimating Systematic Risk in Real-World Networks. In Proceedings of the 18th International Conference on Financial Cryptography and Data Security (FC 2014).

M. Lelarge. 2009. Economics of malware: Epidemic risks model, network externalities and incentives. In Proceedings of the 47th Annual Allerton Conference on Communication, Control, and Computing (Allerton 2009). IEEE, 1353-1360.

M. Lelarge and J. Bolot. 2008. A local mean field analysis of security investments in networks. In Proceedings of the 3rd International Workshop on Economics of Networked Systems (NetEcon 2008). ACM, 25-30.

K. Levchenko, N. Chachra, B. Enright, C. Felegyhazi, M. Grier, T. Halvorson, C. Kanich, C. Kreibich, H. Liu, A. McCoy, D. Pitsillidis, , N. Weaver, V. Paxson, G. M. Voelker, and S. Savage. 2011. Click Trajectories: End-to-End Analysis of the Spam Value Chain. In Proceedings of the 32nd IEEE Symposium on Security and Privacy (Oakland 2011). 431-446.

M. Manshaei, Q. Zhu, T. Alpcan, T. Basar, and J.P. Hubaux. 2013. Game theory meets network security and privacy. Comput. Surveys 45, 3 (2013).

A. Mas-Colell, M.D. Whinston, and J.R. Green. 1995. Microeconomic Theory. Oxford University Press, USA.

D. Meier, Y.A. Oswald, S. Schmid, and R. Wattenhofer. 2008. On the windfall of friendship: Inoculation strategies on social networks. In Proceedings of the 9th ACM Conference on Electronic Commerce (EC 2008). ACM, 294-301.

Microsoft. 2011. Microsoft Security Intelligence Report. Technical Report Vol 12.

Piet Van Mieghem, Jasmina Omic, and Robert E. Kooij. 2009. Virus spread in networks. IEEE / ACM Transactions on Networking 17, 1 (2009), 1-14.

R.A. Miura-Ko, B. Yolken, N. Bambos, and J. Mitchell. 2008a. Security investment games of interdependent organizations. In Proceedings of the 46th Annual Allerton Conference on Communication, Control, and Computing (Allerton 2008). IEEE, 252-260.

R. Miura-Ko, B. Yolken, J. Mitchell, and N. Bambos. 2008b. Security decision-making among interdependent organizations. In Proceedings of the 21st IEEE Computer Security Foundations Symposium (CSF 2008). 66-80.

T. Moore and R. Clayton. 2008. The consequence of non-cooperation in the fight against phishing. In eCrime Researchers Summit. IEEE, 1-14.

T. Moscibroda, S. Schmid, and R. Wattenhofer. 2006. When selfish meets evil: Byzantine players in a virus inoculation game. In Proceedings of the 25th ACM Symposium on Principles of Distributed Computing (PODC 2006). ACM, 35-44.

H. Ogut, N. Menon, and S. Raghunathan. 2005. Cyber insurance and IT security investment: Impact of interdependent risk. In 4th Workshop on the Economics of Information Security (WEIS 2005).

J. Omic, A. Orda, and P. Van Mieghem. 2009. Protecting against network infections: A game theoretic perspective. In Proceedings of the 28th IEEE Conference on Computer Communications (INFOCOM 2009). IEEE, 1485-1493.

Andy Ozment and Stuart E. Schechter. 2006. Bootstrapping the Adoption of Internet Security Protocols.. In 5th Workshop on Economic of Information Security (WEIS 2006).

R. Pal and P. Hui. 2011. Modeling Internet Security Investments: Tackling Topological Information Uncertainty. Proceedings of the 2nd Conference on Decision and Game Theory for Security (GameSec 2011) (2011), 239-257.

S. Radosavac, J. Kempf, and U.C. Kozat. 2008. Using insurance to increase internet security. In Proceedings of the 3rd International Workshop on Economics of Networked Systems (NetEcon 2008). ACM, 43-48.

W. Saad, T. Alpcan, T. Basar, and A. Hjorungnes. 2010. Coalitional game theory for security risk management. In Proceedings of the 5th International Conference on Internet Monitoring and Protection (ICIMP 2010). IEEE, 35-40.

S. Sinha, M. Bailey, and F. Jahanian. 2008. Shades of Grey: On the effectiveness of reputation-based "blacklists". In Proceedings of the 3rd International Conference on Malicious and Unwanted Software (MALWARE 2008). IEEE, 57-64.

G. Stoltz and G. Lugosi. 2007. Learning correlated equilibria in games with compact sets of strategies. Games and Economic Behavior 59, 1 (2007), 187-208.

G. Theodorakopoulos, J.Y.L. Boudec, and J.S. Baras. 2013. Selfish response to epidemic propagation. IEEE Trans. Automat. Control 58, 2 (February 2013), 363-376. 
Hal Varian. 2004. System Reliability and Free Riding. In Economics of Information Security, L.Jean Camp and Stephen Lewis (Eds.). Advances in Information Security, Vol. 12. Springer, 1-15.

Received Month Year; revised Month Year; accepted Month Year 